\begin{tabular}{|c|c|}
\hline JUL 1999 & $\begin{array}{r}\text { Pago } 1 \text { of } \frac{1}{19} \\
8 t a\end{array} 15$ \\
\hline
\end{tabular}






\title{
Master Equipment List 500 CFM Portable Exhauster POR-005 Skid C
}

\author{
J. R. Kriskovich
}

Lockheed Martin Hanford Co., Richland, WA 99352

U.S. Department of Energy Contract DE-AC06-96RL13200

$\begin{array}{llll}\text { EDT/ECN: } & 626839 & \text { UC: } 2000 & \\ \text { Org Code: } & \text { LK500000 } & \text { Charge Code: } 103361 / \text { EF00 } & \text { HN920501 } \\ \text { B\&R Code: } & \text { EW3120071 } & \text { Total Pages: } 13 & \end{array}$

Key Words: $500 \mathrm{cfm}$ exhauster, Master Equipment List

Abstract: The Master Equipment List (MEL) 1ists all the major components of the $500 \mathrm{cfm}$ exhauster POR05. The purpose of this Master Equipment List is to provide basic information and references to other documents for the listed components. In addition to al1 major components, all other identifiable components have been listed. This supporting document has been prepared to make the $500 \mathrm{cfm}$ exhauster "Master Equipment List" readily retrievable.

TRADEMARK DISCLAIMER. Reference herein to any specific comercial product, process, or service by trade name, trademark, manufacturer, or otherwise, does not necessarily constitute or imply its endorsement, recommendation, or favoring by the United States Government or any agency thereof or its contractors or subcontractors.

Printed in the United States of America. To obtain copies of this document, contact: WHC/BCS Document Control Services, P.0. Box 1970, Mailstop H6-08, Richland WA 99352, Phone (509) 372-2420; Fax (509) 376-4989.
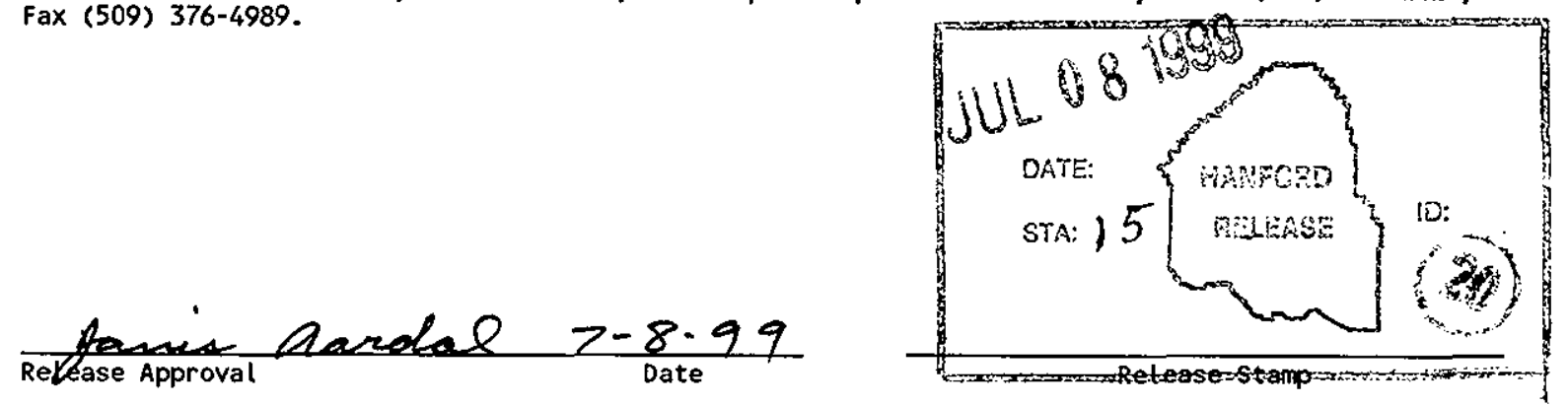
Rev 0

\begin{tabular}{|c|c|c|c|c|c|c|c|c|c|}
\hline EIN & DESCRIPTION & LOCATION & $\begin{array}{l}\text { MANUFACTURER / } \\
\text { MODEL NUMBER }\end{array}$ & SPECIFICATION & $\begin{array}{c}\text { CVI } \\
\text { SUPPLEMENT }\end{array}$ & DRAWING & $\begin{array}{l}\text { MAINTENANCE } \\
\text { PROCEDURE }\end{array}$ & $\begin{array}{l}\text { SAFETY } \\
\text { CLASS }\end{array}$ & $\begin{array}{l}\text { SPARE } \\
\text { PARTS }\end{array}$ \\
\hline POR05-VTP-BKR-101 & $\begin{array}{l}\text { DISTRIBUTION PANEL / VTP-PNL- } \\
101\end{array}$ & PORO5 EXHAUST SKID & SQUARE D/ MPZ7S4DF & $\overline{\mathrm{NA}}$ & NA & $\begin{array}{l}\mathrm{H}-14-100868-1 \mathrm{H}-14- \\
100869 \text { SHT } 1 \text { item \# } 1\end{array}$ & NA & GS & NA \\
\hline POR05.VTP.CON-206 & GLYCOL HEATER O01 & POR05 EXHAUST SKID & $\begin{array}{l}\text { ALLEN-BRADLEY / 500L- } \\
\text { A0093 }\end{array}$ & $\overline{\mathrm{NA}}$ & NA & $\begin{array}{l}\text { H-2.829116 SHT } 1 \text { H-14- } \\
100870 \text { SHT } 2 \text { item } \# 77\end{array}$ & $\overline{N A}$ & GS & $\overline{N A}$ \\
\hline POR05-VTP-CON-301 & SAMPLE VACUUM PUMP STARTER & PRO04 EXHAUST SKID & $\begin{array}{l}\text { TELEMECANIQUE/LC1-D } \\
2510 / \text { LR2D13 }\end{array}$ & NA & NA & $\begin{array}{l}\mathrm{H}-14-100916 \mathrm{SHT} 1.5 \\
\text { item \# 46/478 }\end{array}$ & NÁ & GS & $\overline{\mathrm{NA}}$ \\
\hline POR05-VTP-CON-302 & SAMPLE VACUM PUMP STARTER & POR05 EXHAUST SKID & $\begin{array}{l}\text { TELEMECANIQUE / LCI- } \\
02510\end{array}$ & NA & NA & $\begin{array}{l}\mathrm{H}-14-100916 \mathrm{SHT} 1,5 \\
\text { item \# 46/47 }\end{array}$ & NA & GS & NA \\
\hline POR05-VTP-CONV-101 & $\begin{array}{l}\text { 485 DH DATA HIGHWAY MODULE } \\
\text { OATA LINK COUPLER/SCL } 500 \\
\text { AND CABLE }\end{array}$ & POR05 EXHAUST SKID & $\begin{array}{l}\text { ALLEN 8RAOLEY / } 1747- \\
\text { ALC, 1747.C11 }\end{array}$ & $\overline{N A}$ & NA & $\begin{array}{l}\text { H-14-100870 SHT } 2 \text { ITEM } \\
\# 142,143 \text { H-2-829116 } \\
\text { SHT } 1\end{array}$ & NA & GS & NA \\
\hline POR05.VTP-CP.105 & EXHAUST CONTROL PANEL & PORO5 EXHAUST SKID & HOFFMAN/A-36H30FLP & NA & NA & $\begin{array}{l}\mathrm{H}-2-829116 \text { SHT } 1, \mathrm{H}-14- \\
100870 \text { SHT } 1,3 \text { item \# } \\
21\end{array}$ & NA & GS & NA \\
\hline PORO5-VTP-DS-101 & $\begin{array}{l}\text { PORTABLE EXKAUSTER SKIO } \\
\text { MAIN POWER }\end{array}$ & PORO5 EXHAUST SKID & $\begin{array}{l}\text { WESTINGHOUSENFDN } \\
100, \text { EHD3030 }\end{array}$ & NA & NA & $\begin{array}{l}H \cdot 14-100868-1 \text { H-14- } \\
100870 \text { SHT } 1 \text { item } \# 1,2\end{array}$ & $\overline{N A}$ & GS & NA \\
\hline POR05-VTP.DS-102 & $\begin{array}{l}\text { EXHAUST FAN MOTOR } 001 \\
\text { SUPPLY FUSED DISCONNECT } \\
\text { SWITCH }\end{array}$ & POROS EXHAUST SKIO & $\begin{array}{l}\text { CUTLER-HAMMER } \\
\text { DH361FRK BUSSMAN } \\
\text { NOS.6 }\end{array}$ & $\mathrm{NA}$ & NA & \begin{tabular}{|l|}
$\mathrm{H}-14-100868-1$ \\
100870 SHT 1 ITEM $\# 7,8$
\end{tabular} & NA & GS & $\overline{N A}$ \\
\hline POR05-VTP.DS-201 & $\begin{array}{l}\text { GLYCOL HEATER MOTOR O01 } \\
\text { SUPPLYY FUSED DISCONNECT } \\
\text { SWITCH }\end{array}$ & POROS EXHAUST SKID & $\begin{array}{l}\text { CUTLER-HAMMER } \\
\text { DH361FRK BUSSMAN } \\
\text { NOS-10 }\end{array}$ & NA & NA & $\mid \begin{array}{l}\mathrm{H}-14-100868-1 \\
100870 \text { SHT } 1\end{array}$ & $\overline{N A}$ & GS & $\overline{N A}$ \\
\hline POR05-VTP.EF-001 & VENT TRAIN EXHAUST FAN & POROS EXHAUST SKIO & $\begin{array}{l}\text { BARRYBLOWER /7.16 } \\
\text { TYPE OT-15 }\end{array}$ & $\overline{\mathrm{NA}}$ & $\begin{array}{c}22758 \text { ITEM \# } 7 \\
13,15\end{array}$ & \begin{tabular}{|l|}
$\mathrm{H}-2-829116$ SHT $1 \mathrm{H}-14$. \\
100867 SHT 1 see note \#1
\end{tabular} & 6-TF-237 & GS & $\overline{N A}$ \\
\hline POR05-VTP-ENCL-104 & HEAT TRACE CABINET & POROS EXHAUST SKID & HOFFMAN/A-30P2O & $\overline{\mathrm{NA}}$ & 22758 ITEM \# 38 & \begin{tabular}{|l|} 
H-2-829116 SHT 2 H-14- \\
100870 SHT 2 ITEM \# 51
\end{tabular} & $\mathrm{NA}$ & GS & $\overline{N A}$ \\
\hline POR05-VTP-ENCL-106 & INTRINSIC BARRIER CABINET & POR05 EXHAUST SKID & $\begin{array}{l}\text { ELECTROMATE/ELME2O } \\
\mathrm{H} 20 \mathrm{~B}\end{array}$ & NA & NA & $\begin{array}{l}\mathrm{H}-14-829116-5 \mathrm{H}-14 \\
100870 \text { SHT } 2 \text { ITEM \# 110 }\end{array}$ & $\overline{\mathrm{NA}}$ & GS & NA \\
\hline POR05-VTP-ENCL-107 & EXHAUSTER ALARM PANEL & POR05 EXHAUST SKID & HOFFMAN, C-SD242416 & NA & $\mathrm{NA}$ & \begin{tabular}{|l|}
$\mathrm{H}-2-829116$ SHT $1, \mathrm{H}-14-$ \\
$100870 \mathrm{SHT} 1,5$ TTEM $\#$ \\
15
\end{tabular} & $\overline{\mathrm{NA}}$ & GS & $\overline{N A}$ \\
\hline POR05-VTP-ENCL-301 & SAMPLE CABINET ENCLOSURE & POROS EXHAUST SKID & $\begin{array}{l}\text { HOFFMAN/A- } \\
36 \mathrm{H} 3016 S S L P\end{array}$ & $\overline{N A}$ & NA & \begin{tabular}{|l|}
$\mathrm{H}-2-829116$ SHT $5 \mathrm{H}-14-$ \\
100916 SHT 1,4 ITEM \# 2
\end{tabular} & NA & GS & NA \\
\hline POR05-VTP-ENCL-302 & PUMP CABINET ENCLOSURE & POR05 EXHAUST SKID & $\begin{array}{l}\text { HOFFMAN/A. } \\
\text { 36H3016SSLP }\end{array}$ & $\mathrm{NA}$ & $\overline{\mathrm{NA}}$ & $\begin{array}{l}\mathrm{H} \cdot 2 \cdot 829116 \text { SHT } 5 \text { H-14. } \\
100916 \text { SHT 1, } 5 \text { ITEM \# } \\
42\end{array}$ & NA & $\overline{G S}$ & $\overline{N A}$ \\
\hline POR05-VTP.F-104 & $\begin{array}{l}\text { HEAT TRACE CABINET } 104 \\
\text { VENTILATION FAN }\end{array}$ & POROS EXKAUST SKIO & HOFFMAN/A-4AXFN & NA & NA & $\begin{array}{l}\text { H-2-829116 SHT 2H-14- } \\
100870 \text { SHT } 1 \text { ITEM \# } 40\end{array}$ & NA & GS & NA \\
\hline POR0S-VTP-F-10S & $\begin{array}{l}\text { EXHAAUSTER CONTROL PANEL } \\
\text { VENT FAN } 105\end{array}$ & POROS EXXHAUST SKID & HOFFMAN/A-PA10AXFN & NA & NA & \begin{tabular}{|l|} 
H-2-829116 SHT 2 H-14- \\
I00870 SHT 1 ITEM \# 37
\end{tabular} & $\overline{N A}$ & GS & $\mathrm{NA}$ \\
\hline POR05-VTP-F-106 & INTRINSIC BARRIER CABINET & FOR05 EXHAUST SKIO & Electromate / Elme20h20b & $\overline{N A}$ & $\overline{N A}$ & $\begin{array}{l}H-2-829116 \text { SHT } 2 \mathrm{H}-14- \\
100870 \text { SHT } 1 \text { ITEM } \# \text { 110 }\end{array}$ & NA & GS & NA \\
\hline
\end{tabular}




\begin{tabular}{|c|c|c|c|c|c|c|c|c|c|}
\hline EIN & DESCRIPTION & LOCATION & $\begin{array}{l}\text { MANUFACTURER I } \\
\text { MODEL NUMBER }\end{array}$ & SPECIFICATION & $\begin{array}{c}\text { CVI } \\
\text { SUPPLEMENT }\end{array}$ & DRAWING & $\begin{array}{l}\text { MAINTENANCE } \\
\text { PROCEDURE }\end{array}$ & $\begin{array}{l}\text { SAFETY } \\
\text { CLASS }\end{array}$ & $\begin{array}{l}\text { SPARE } \\
\text { PARTS }\end{array}$ \\
\hline POR05-VTP-F-107 & ALARM PANEL 107 VENT FAN & POR05 EXHAUST SKIO & HOFFMAN/A-4AXFN & NA & NA & $\begin{array}{l}\mathrm{H}-2-829116 \text { SHT } 2 \text { H-14. } \\
100870 \text { SHT } 2,5 \text { ITEM \# } \\
40\end{array}$ & NA & GS & NA \\
\hline POR05.VTP-F-301 & SAMPLE CABINET 301 VENT FAN & POR05 EXHAUST SKID & HOFFMAN/A.4AXFN & NA & NA & $\begin{array}{l}\text { H-2-829116 SHT } 5 \text { H-14- } \\
100916 \text { SHT } 1 \text {, } 4 \text { ITEM \# } \\
5\end{array}$ & NA & GS & $\overline{N A}$ \\
\hline POR05-VTP-F.302 & PUMP CABINET 302 VENT FAN & POR05 EXHAUST SKID & HOFFMAN/A-6AXFN & NA & $\mathrm{NA}$ & $\begin{array}{l}\text { H-2-829116 SHT } 5 \text { H-14- } \\
100916 \text { SHT 1, } 5 \text { ITEM \# } \\
49\end{array}$ & NA & GS & $\overline{N A}$ \\
\hline POR05-VTP-FCV-301 & $\begin{array}{l}\text { EXHAUST STACK RECORD } \\
\text { SAMPLE FLOW CONTROL VALVE }\end{array}$ & PORO5 EXHAUST SKID & HASTINGS/MFC.203 & NA & 22758 ITEM \# 43 & $\begin{array}{l}\mathrm{H}-2-829116 \text { SHT } 5 \text { H-14. } \\
00916-1,4 \text { ITEM \# } 4\end{array}$ & $6-\mathrm{FCD}-521$ & GS & $\overline{N A}$ \\
\hline POR05-VTP.FCV-302 & $\begin{array}{l}\text { EXHAUST STACK CAM SAMPLE } \\
\text { LINE FLOW CONTROL VALVE }\end{array}$ & POROS EXHAUST SKID & HASTINGS/HFC-203 & $\overline{\mathrm{NA}}$ & 22758 ITEM \# 43 & $\begin{array}{l}\mathrm{H}-2-829116 \text { SHT } 5 \text { H-14- } \\
100916-1,4 \text { ITEM \# } 4\end{array}$ & $6 \cdot F C D-521$ & SS & $\mathrm{NA}$ \\
\hline POR05-VTP-FE-184 & EXHAUST STACK FLOW ELEMENT & POR05 EXHAUST SKID & $\begin{array}{l}\text { VERIS VERABAR I V100- } \\
6,40-05-\mathrm{V}-\mathrm{T}\}\end{array}$ & NA & $\begin{array}{c}22758 \text { ITEM \# 40, } \\
41\end{array}$ & $\begin{array}{l}\text { H-2-829116 SHT 1 H-14- } \\
100867 \text { SHT } 1,9 \text { Item \# } \\
18\end{array}$ & NA & GS & NA \\
\hline POR05-VTP-FI-184 & $\begin{array}{l}\text { EXHAUST STACK FLOW } \\
\text { INDICATOR }\end{array}$ & POR0S EXHAUST SKID & $\begin{array}{l}\text { WILKERSON/ DIS47\%- } \\
\text { 24-115-4/2OMA }\end{array}$ & $\overline{N A}$ & 22758 ITEM \# 36 & \begin{tabular}{|l}
$\mathrm{H}-2-829116-1 \mathrm{H}-14-$ \\
$100870-1,4$ ITEM $\# 23$
\end{tabular} & 6-GENI-135 & GS & NA \\
\hline POR05-VTP-FLT.001 & EXHAUST TRAIN PRE FILTER & PORO5 EXHAUST SKID & \begin{tabular}{|l|} 
FARR / UL CLASS 1, \\
30/30, $24^{\prime \prime} \times 24^{\prime \prime} \times 2 "$, \\
FARR PART \#074181005
\end{tabular} & $\overline{\mathrm{NA}}$ & 22758 ITEM \# 8 & $\begin{array}{l}\text { H-2-829116 SHT } 1 \text { H-14. } \\
100867 \text { SHT } 2,6 \text { see note } \\
\# 3 \text { item } \# 25,30\end{array}$ & NA & GS & YES \\
\hline POR05-VTP-FLT-002 & EXHAUST TRAIN 1ST HEPA FILTER & POROS EXHAUST SKID & $\begin{array}{l}\text { FLANDERS / PART \#T- } \\
\text { O07-D-03-05-NU-51-23- } \\
\text { GG-DU5 or T-007-D-43- } \\
\text { 05-NU-51-23-GG-DU5 }\end{array}$ & NA & \begin{tabular}{|c|}
22758 ITEM \# 20, \\
$21,22,23,24$, \\
$25,26,42$
\end{tabular} & \begin{tabular}{|l|}
$\mathrm{H}-2-829116 \mathrm{SHT}$ \\
$100867 \mathrm{H}-14-$ \\
$\# 3$
\end{tabular} & $6-T F-156 T X$ & GS & YES \\
\hline POR05-VTP-FLT-003 & $\begin{array}{l}\text { EXHAUST TRAIN 2ND HEPA } \\
\text { FILTER }\end{array}$ & POR05 EXHAUST SKIO & $\begin{array}{l}\text { FLANDERS / PART \#T- } \\
\text { O07-O-03-05-NU-51-23- } \\
\text { GG-DU5 OR t-007-d-43- } \\
\text { 05-NU-51-23-GG-DU5 }\end{array}$ & NA & $\begin{array}{c}22758 \text { ITEM } \# 20, \\
21,22,23,24, \\
25,26,42\end{array}$ & $\begin{array}{l}\mathrm{H}-2 \cdot 829116 \text { SHT } 1 \mathrm{H}-14- \\
100867 \text { SHT } 2,6 \text { see note } \\
\# 3\end{array}$ & 6-TF-156TX & GS & YES \\
\hline PORO5-VTP.FLT-101 & TANK S-102 BREATHER FILTER & TANK S-102 & $\begin{array}{l}\text { FLANDERS / PART \#T- } \\
\text { O07-D-03-05-NU-51-23- } \\
\text { GG-DU5 OR t-007-d-43- } \\
\text { 05-NU-51-23-GG-DU5 }\end{array}$ & HNF-S.0552 & $\begin{array}{c}22758 \text { ITEM \# } 20 \\
21,22,23,24 \\
25,26,42\end{array}$ & H-2-73182 SHT 1 & 6-TF-156SB & $\overline{G S}$ & YES \\
\hline POR05-VTP-FLT-102 & TANK S.103 BREATHER FILTER & TANK S-103 & $\begin{array}{l}\text { FLANDERS / PART \#T. } \\
\text { O07-D-03-05-NU-51-23- } \\
\text { GG-DU5 OR t-007-d-43- } \\
\text { 05-NU-51-23-GG-DU5 }\end{array}$ & HNF-S-0552 & $\mid \begin{array}{c}22758 \text { ITEM \# } 20, \\
21,22,23,24 \\
25,26,42\end{array}$ & $\mathrm{H}-2-73182 \mathrm{SHT} 1$ & 6-TF-156SB & GS & YES \\
\hline PORO5-VTP-FLT-103 & TANK S.016 BREATHER FILTER & TANK S-016 & $\begin{array}{l}\text { FLANDERS / PART HT- } \\
\text { O07-D-03-05-NU-51-23- } \\
\text { GG-DU5 OR t-007-d-43- } \\
\text { 05-NU-51-23-GG-DU5 }\end{array}$ & HNF-S-0552 & $\begin{array}{c}22758 \text { ITEM \# } 20 \\
21,22,23,24 \\
25,26,42\end{array}$ & $\mathrm{H}-2.73182 \mathrm{SHT} 1$ & 6-TF-156SB & GS & YES \\
\hline POR05-VTP-FLT-301 & RECORD SAMPLE FILTER & POR05 EXHAUST SKID & $\begin{array}{l}\text { GELMAN SCIENCES } \\
\$ 2200\end{array}$ & NA & NA & $\begin{array}{l}\text { H-14-100916 SHT 1,4 } \\
\text { item \# } 12\end{array}$ & NA & GS & NA \\
\hline POR05-VTP-FT-184 & EXHAUST STACK FLOW XMTR & POR05 EXHAUST SKID & $\begin{array}{l}\text { YOKOGAWAVEJA120- } \\
\text { DES 4B-92EC/FS } 1 / 01\end{array}$ & NA & 22758 ITEM \# 32 & $\begin{array}{l}\text { H-2-829116 SHT } 1 \mathrm{H}-14- \\
100869-1,3 \text { item } \# 21\end{array}$ & 6-PCD-373 & SS & YES \\
\hline
\end{tabular}




\begin{tabular}{|c|c|c|c|c|c|c|c|c|c|}
\hline EIN & DESCRIPTION & LOCATION & $\begin{array}{l}\text { MANUFACTURER } / \\
\text { MODEL NUMBER }\end{array}$ & SPECIFICATION & $\begin{array}{c}\text { CV! } \\
\text { SUPPLEMENT }\end{array}$ & DRAWING & $\begin{array}{l}\text { MAINTENANCE } \\
\text { PROCEDURE }\end{array}$ & $\begin{array}{l}\text { SAFETY } \\
\text { CLASS }\end{array}$ & $\begin{array}{l}\text { SPARE } \\
\text { PARTS }\end{array}$ \\
\hline POR05-VTP.HS.101 & $\begin{array}{l}\text { SEAL POT PUMP } 004 \text { ON/OFF } \\
\text { SWITCH }\end{array}$ & POR05 EXHAUST SKID & $\begin{array}{l}\text { ALLEN-BRADLEY/800T- } \\
\text { H42A }\end{array}$ & NA & NA & $\begin{array}{l}\text { H-2-829116 SHT } 1 \text { H-14- } \\
100870 \text { SHT } 1,4 \text { ITEM \# } \\
13\end{array}$ & NA & GS & NA \\
\hline POR05-VTP-HS-102 & $\begin{array}{l}\text { GLYCOL PUMP } 001 \text { ON/OFF } \\
\text { SWITCH }\end{array}$ & POR05 EXHAUST SKID & $\begin{array}{l}\text { ALLEN-BRADLEY/800T- } \\
\text { H17D1 X559E }\end{array}$ & NA & NA & $\begin{array}{l}\text { H-2.829116 SHT } 1 \text { H-14- } \\
100870 \text { SHT 2, } 4 \text { ITEM \# } \\
93\end{array}$ & NA & GS & NA \\
\hline POR05-VTP.HS-103 & $\begin{array}{l}\text { EXHAUST FAN } 001 \text { OFF/ENABLE } \\
\text { SWITCH }\end{array}$ & POR05 EXYAUST SKID & $\begin{array}{l}\text { ALLEN-BRADLEY/800T- } \\
\text { H1701 }\end{array}$ & NA & NA & $\begin{array}{l}\mathrm{H}-2-829116 \text { SHT } 1 \text { H-14- } \\
100870 \text { SHT } 1,4 \text { ITEM \# } \\
11\end{array}$ & NA & GS & NA \\
\hline POR05-VTP-HS-104 & $\begin{array}{l}\text { ENCLOSURE } 104 \text { AND } 106 \\
\text { SUMMERMINTER CONTROL SW }\end{array}$ & POR05 EXHAUUST SKID & $\begin{array}{l}\text { ALLEN-BRADLEY/800T- } \\
\text { J17A }\end{array}$ & $\overline{\mathrm{NA}}$ & NA & $\begin{array}{l}\text { H-14-100868 SHT } 5 \text { H-14 } \\
100870 \text { SHT } 2,4 \text { ITEM \# } \\
96,97\end{array}$ & NA & GS & $\overline{\mathrm{NA}}$ \\
\hline POR05-VTP-HS-105 & $\begin{array}{l}\text { ENCLOSURE } 105 \text { AND } 107 \\
\text { SUMMERMINTER CONTROL SW }\end{array}$ & POROS EXHAUST SKID & $\begin{array}{l}\text { ALLEN-BRADLEY/800T- } \\
\text { J17A }\end{array}$ & $\overline{N A}$ & $\overline{N A}$ & \begin{tabular}{|l|}
$\mathrm{H}-14-100868$ SHT 5 H-14 \\
$100870-2,4$ ITEM $\# 96$, \\
97
\end{tabular} & NA & GS & NA \\
\hline POR05-VTP-HTR-001 & GLYCOLL HEATER & POR05 EXHAUST SKID & $\begin{array}{l}\text { CHROMALOX / NWHMT- } \\
3605 E 2\end{array}$ & NA & \begin{tabular}{|l|}
22758 ITEM \# 29 \\
\end{tabular} & \begin{tabular}{|l|} 
H-2-829116 SHT 1 H-14- \\
100867 SHT 3, 18 ITEM \# \\
136 \\
\end{tabular} & NA & GS & NA \\
\hline POR05-VTP-HTR-104 & $\begin{array}{l}\text { HEAT TRACE CABINET } 104 \\
\text { HEATER }\end{array}$ & POR05 EXHAUST SKID & HOFFMAN/D-AH1001A & NA & NA & \begin{tabular}{|l|} 
H-2.829116 SHT 2, H-14- \\
100870 SHT 1, 3 ITEM \# \\
39 \\
\end{tabular} & NA & GS & NA \\
\hline POR05-VTP-HTR-105 & $\begin{array}{l}\text { EXHAUSTER CONTROL PANEL } \\
105 \text { HEATER }\end{array}$ & POR05 EXHAUST SKID & HOFFMAN/D-AH2001A & NA & $\overline{N A}$ & $\begin{array}{l}\text { H-2-829116 SHT 2, H-14- } \\
100870 \text { SHT 1, } 3 \text { ITEM \# } \\
38\end{array}$ & NA & GS & NA \\
\hline POR05-VTP-HTR-106 & $\begin{array}{l}\text { INTRINSIC BARRIER CABINET } 106 \\
\text { HEATER }\end{array}$ & POR05 EXHAUST SKID & HOFFMAN / D-AH1001A & NA & $\overline{N A}$ & $\begin{array}{l}\text { H-2-829116 SHT H-4- } \\
100870 \text { SHT 1, 3 ITEM \# } \\
39\end{array}$ & $\overline{N A}$ & GS & NA \\
\hline POR05-VTP.HTR-107 & ALARM PANEL 107 HEATER & POR05 EXHAUST SKID & HOFFMAN/C-SO242416 & $\mathrm{NA}$ & $\overline{N A}$ & $\begin{array}{l}\text { H-2-829116 SHT } 2 \text { H-14- } \\
100870 \text { SHT } 1,4 \text { ITEM \# } \\
39\end{array}$ & NA & GS & $\overline{\mathrm{NA}}$ \\
\hline POR05.VTP.HTR-301 & SAMPLE CABINET 301 HEATER & POR05 EXHAUST SKID & ROFFMAN/D-AH40018 & NA & $\overline{N A}$ & $\begin{array}{l}\text { H-2-829116 SHT } 5 \text { H-14- } \\
100916 \text { SHT } 1,4 \text { ITEM \# } \\
11\end{array}$ & NA & GS & NA \\
\hline POR05-VTP-HTR-302 & PUMP CABINET 302 HEATER & POR05 EXHAUST SKIO & HOFFMAN/D-AH2001A & NA & \begin{tabular}{|l|} 
NA \\
\end{tabular} & $\begin{array}{l}\text { H-2-829116 SHT } 5 \text { H-14- } \\
100916 \text { SHTT 1, } 5 \text { ITEM \# } \\
48\end{array}$ & NA & GS & NA \\
\hline POR05-VTP-HX-001 & $\begin{array}{l}\text { VENT EXYAUUST TRAIN INLET } \\
\text { HEAT EXCHANGER }\end{array}$ & PORO5 EXHAUST SKID & $\begin{array}{l}\text { EXCHANGER, INC/ } \\
\text { OWG } 22444\end{array}$ & NA & 22758 ITEM \# 28 & \begin{tabular}{|l|}
$\mathrm{H}-2-829116$ SHT $1 \mathrm{H}-14-$ \\
100867 SHT 3,18 ITEM \# \\
137
\end{tabular} & NA & GS & NA \\
\hline POR05-VTP-LG-201 & $\begin{array}{l}\text { GLYCOL EXPANSION TANK } 001 \\
\text { LEVEL GAUGE }\end{array}$ & POR05 EXHAUST SKID & $\begin{array}{l}\text { MCMASTER-CARR-96/ } \\
\text { 1106KA }\end{array}$ & $\overline{N A}$ & NA & \begin{tabular}{|l|}
$\mathrm{H}-2-829116-1, \mathrm{H}-14$. \\
$100867-3,18$ ITEM \# 147
\end{tabular} & NA & GS & NA \\
\hline POR05-VTP-LI-185 & SEAL POT 001 LEVEL INDICATOR & POR05 EXHAUUST SKID & $\begin{array}{l}\text { WILKERSON/DIS } 471.24 \\
\text { G.115-4/2OMA }\end{array}$ & NA & 22758 ITEM \# 36 & $\begin{array}{l}\text { H-2-829116 SHT } 1 \text { H-14- } \\
100870 \text { SHT } 1,4 \text { ITEM \# } \\
23\end{array}$ & 6-GENI-135 & GS & NA \\
\hline POR05-VTP-LI-205 & $\begin{array}{l}\text { GLYCOL EXPANSION TANK } 001 \\
\text { LEVEL INDICATOR }\end{array}$ & POR05 EXHAUST SKID & \begin{tabular}{|l|} 
WILKERSON/DIS 471-24 \\
G-115-4/2OMA
\end{tabular} & NA & 22758 ITEM \# 36 & $\begin{array}{l}\text { H-2-829116 SHT } 1 \text { H-14- } \\
100870 \text { SHT } 1,4 \text { ITEM \# } \\
23\end{array}$ & 6. GENI-135 & GS & NA \\
\hline
\end{tabular}




\begin{tabular}{|c|c|c|c|c|c|c|c|c|c|}
\hline EIN & DESCRIPTION & LOCATION & $\begin{array}{l}\text { MANUFACTURER / } \\
\text { MODEL NUMBER }\end{array}$ & SPECIFICATION & $\begin{array}{c}\text { CVI } \\
\text { SUPPLEMENT }\end{array}$ & DRAWING & $\begin{array}{l}\text { MAINTENANCE } \\
\text { PROCEDURE }\end{array}$ & $\begin{array}{l}\text { SAFETY } \\
\text { CLASS }\end{array}$ & $\begin{array}{l}\text { SPARE } \\
\text { PARTS }\end{array}$ \\
\hline PORO5-VTP-LT-185 & SEAL POT 001 LEVEL XMTR & POR05 EXHAUST SKID & $\begin{array}{l}\text { OREXELEROOK/CS08-45 } \\
709\end{array}$ & NA & 22758 ITEM \# 39 & $\begin{array}{l}\text { H-2-829116 SHT } 1 \text { H-14- } \\
100869-1,3 \text { ITEM \# } 27\end{array}$ & 6 -CVT-331 & GS & NA \\
\hline POR05-VTP-LT-205 & $\begin{array}{l}\text { GLYCOOL EXPANSION TANK } 001 \\
\text { LEVEL XMTR }\end{array}$ & POR05 EXHAUST SKID & $\begin{array}{l}\text { DREXELBROOKS/CSO8- } \\
45.9\end{array}$ & $\overline{N A}$ & 22758 ITEM \#39 & $\begin{array}{l}\text { H-2-829116 SHT } 1 \text { H-14- } \\
100869-1,3 \text { ITEM } \# 26\end{array}$ & 6-cVT-331 & GS & NA \\
\hline PORO5-VTP-M-001 & EXHAUST FAN MOTOR & POR05 EXHAUST SKID & $\begin{array}{l}\text { SEE NOTE \# } 1 \mathrm{H}-14 \cdot \\
100867 \mathrm{SHT} 2\end{array}$ & NA & 22758 ITEM \# 39 & $\begin{array}{l}\text { H-2-829116 SHT } 1 \text { H-14- } \\
\text { 100869-2, NOTE \# } 1\end{array}$ & NA & GS & NA \\
\hline POR05-VTP-MT-101 & $\begin{array}{l}\text { EXHAUST STACK INLET } \\
\text { MOISTURE XMTR }\end{array}$ & POR05 EXHAUST SKID & HY-CALCT-880-CH12 & NA & 22758 ITEM $\# 50$ & $\begin{array}{l}\text { H-2-829116 SHT } 1 \mathrm{H}-14- \\
100869-1,3 \text { ITEM } \# 38\end{array}$ & NA & GS & NA \\
\hline POR05-VTP-P-DO1 & GLYCOL RECIRCULATING PUMP & POR05 EXHAUST SKIO & $\begin{array}{l}\text { LAING THERMOTECH, } \\
\text { INC / SM-9OS-8F }\end{array}$ & $\overline{N A}$ & $\mathrm{NA}$ & $\begin{array}{l}\text { H-2-829116 SHT } 1 \text { H-14- } \\
100867 \text { SHT 3, } 18 \text { ITEM \# } \\
131\end{array}$ & NA & GS & $\mathrm{NA}$ \\
\hline POR05-VTP.P.004 & SEAL POT 001 PUMP & POROS EXHAUST SKID & $\begin{array}{l}\text { RANDOLPH AUSTIN/ } \\
610-100\end{array}$ & NA & 22758 ITEM \# 46 & $\begin{array}{l}\text { H-2.829116 SHT } 1 \text { H.14- } \\
100867 \text { SHT 5, } 7 \text { ITEM \# } \\
217\end{array}$ & 5-FACE-418 & GS & $\mathrm{NA}$ \\
\hline POR05-VTP.P-301 & SAMPLE VACUUM PUMP & POR05 EXHAUST SKIO & $\begin{array}{l}\text { METAL-BELLLOWS / } \\
\text { MB602 }\end{array}$ & NA & 22758 ITEM \# 45 & $\begin{array}{l}\text { H-2-829116 SHT i H-14- } \\
100916 \text { SHT } 1 \text {, } 5 \text { ITEM \# } \\
44\end{array}$ & NA & GS & $\overline{N A}$ \\
\hline POR05-VTP-P-302 & SAMPLE VACUUM PUMP & PORO5 EXHAUST SKID & $\begin{array}{l}\text { METAL-8ELLOWS / } \\
\text { MB602 }\end{array}$ & NA & 22758 ITEM \# 45 & $\begin{array}{l}\text { H.2-829116 SHT } 1 \text { H-14- } \\
100916 \text { SHT } 1,5 \text { ITEM \# } \\
44\end{array}$ & NA & GS & NA \\
\hline POR05.VTP-PB-101 & $\begin{array}{l}\text { EXHAUST FAN } 001 \text { START PUSH } \\
\text { BUTTON }\end{array}$ & PORO5 EXHAUST SKIO & \begin{tabular}{|l} 
ALLEN-BRAOLEY/800T. \\
B1A
\end{tabular} & NA & NA & $\begin{array}{l}\mathrm{H}-2-829116 \text { SHT } 1 \text { H-14- } \\
100870 \text { SHT } 1,4 \text { ITEM \# } \\
27\end{array}$ & NA & GS & $\overline{N A}$ \\
\hline PORO5-VTP-PB-102 & $\begin{array}{l}\text { EXHAUST FAN } 001 \text { STOP PUSH } \\
\text { BUTTON }\end{array}$ & POR05 EXHAUST SKID & $\begin{array}{l}\text { ALLEN-BRADLEY / 800T- } \\
\text { B6A }\end{array}$ & NA & NA & $\begin{array}{l}\text { H.2-829116-1 H.14. } \\
100870-1,4 \text { ITEM \# } 29\end{array}$ & NA & GS & $\overline{N A}$ \\
\hline POR05-VTP-PB-103 & $\begin{array}{l}\text { EMERGENCY POWER SHUTDOWN } \\
\text { PUSH BUTTON }\end{array}$ & PORO5 EXHAUST SKIO & $\begin{array}{l}\text { ALLEN-BRADLEY / 800T- } \\
\text { D6JA }\end{array}$ & $\overline{\mathrm{NA}}$ & $\overrightarrow{N A}$ & $\begin{array}{l}\text { H-2-829116 SHT I H-14- } \\
100870-1,3 \text { ITEM } \# 4\end{array}$ & $\overline{\mathrm{NA}}$ & GS & $\overline{\mathrm{NA}}$ \\
\hline POR05-VTP-PB-104 & CAM BYPASS PUSH BUTTON & PORO5 EXHAUST SKID & $\begin{array}{l}\text { ALLEN-BRADLEY 800T: } \\
\text { A1A }\end{array}$ & NA & NA & $\begin{array}{l}\text { H-2-829116 SHT } 1 \text { H-14- } \\
100870-2,4 \text { ITEM } \# 121\end{array}$ & NA & GS & $\overline{\mathrm{NA}}$ \\
\hline POR05-VIP.POI-170 & $\begin{array}{l}\text { TANK SOUURE } 1 \text { PLENUM INLET } \\
\text { PRESS DIFF INDICATOR }\end{array}$ & POR05 EXHAUST SKIO & $\begin{array}{l}\text { WILKERSON/DIS } 471-24 \\
\text { G-115-4/20MA }\end{array}$ & NA & 22758 ITEM \# 36 & $\begin{array}{l}\text { H-2-829116 SHT 3 H-14. } \\
100870-1, \text { ITEM } \# 23\end{array}$ & 6-GENI-135 & GS & NA \\
\hline POR05-VTP-PDI-171 & $\begin{array}{l}\text { TANK SOURCE } 3 \text { PLENUM INLET } \\
\text { PRESS DIFF INDICATOR }\end{array}$ & POROS EXHAUST SKIO & $\begin{array}{l}\text { WILKERSON/ DIS471-24 } \\
\text { G-115-4/2OMA }\end{array}$ & NA & 22758 ITEM \# 36 & $\begin{array}{l}\text { H-2-829116 SHT 3 R-14- } \\
100870 \cdot 1,2 \text { ITEM \# } 23\end{array}$ & 6-GENI-135 & GS & NA \\
\hline POR05-VTP-POI-173 & $\begin{array}{l}\text { TANK SOURCE } 2 \text { PLENUM INLET } \\
\text { PRESS OIFF INDICATOR }\end{array}$ & PORO5 EXMAUST SKID & $\begin{array}{l}\text { WILKERSON/OIS471-24 } \\
\text { G-115-4/2OMA }\end{array}$ & $\overline{\mathrm{NA}}$ & 22758 ITEM \# 36 & $\begin{array}{l}\mathrm{H}-2-829116 \text { SHT } 2 \mathrm{H}-14- \\
100870-1,2 \text { ITEM \# } 23\end{array}$ & 6. GENI-135 & GS & NA \\
\hline POR05-VTP-PDI-177 & $\begin{array}{l}\text { HEAT EXCHANGE } 001 \text { PRESS DIFF } \\
\text { INDICATOR }\end{array}$ & POR05 EXHAUST SKID & $\begin{array}{l}\text { WILKERSON/DIS471-24 } \\
\text { G.115-4/2OMA }\end{array}$ & NA & 22758 ITEM \# 36 & $\begin{array}{l}\text { H-2-829116 SHT 1 H-14- } \\
\text { 100870-1, } 2 \text { ITEM \# } 23\end{array}$ & $\overline{\mathrm{NA}}$ & GS & $\overline{N A}$ \\
\hline POR05-VTP-PDI-178 & $\begin{array}{l}\text { PRE FILTER } 001 \text { PRESS DIFF } \\
\text { INDICATOR }\end{array}$ & PORO5 EXHAUST SKID & $\begin{array}{l}\text { WILKERSON/ DIS471-24 } \\
\text { G-315-4/2OMA ITEM }\end{array}$ & NA & 22758 ITEM \# 36 & $\begin{array}{l}\text { H-2-829116 SHT 1 H-14- } \\
100870-1,2 \text { ITEM } \# 23\end{array}$ & NA & GS & NA \\
\hline POR05-VTP-PDI-180 & $\begin{array}{l}\text { 1ST HEPA FILTER O02 PRESS DIFF } \\
\text { INDICATOR }\end{array}$ & PORO5 EXHAUST SKID & $\begin{array}{l}\text { WILKERSON/DIS471-24 } \\
\text { G.115-4/20MA }\end{array}$ & NA & 22758 ITEM \# 36 & $\begin{array}{l}\text { H-2-829116 SHT 1 H-14- } \\
100870-1,2 \text { ITEM \# } 23\end{array}$ & 6.GENt-135 & GS & NA \\
\hline POR05-VTP-PDI-181 & $\begin{array}{l}\text { FILTER TRAIN PRESS DIFF } \\
\text { INDICATOR }\end{array}$ & POR05 EXHAUST SKID & $\begin{array}{l}\text { WLLKERSON/DIS471-24 } \\
\text { G.115-4/2OMA }\end{array}$ & NA & 22758 ITEM \# 36 & $\begin{array}{l}\text { H-2-829116 SHT } 1 \text { H-14- } \\
100870-1,2 \text { ITEM \# } 23\end{array}$ & 6-GENI-135 & GS & NA \\
\hline POR05-VTP-PDI-182 & $\begin{array}{l}\text { 2ND HEPA FILTER } 003 \text { PRESS } \\
\text { DIFF INDICATOR }\end{array}$ & POR05 EXHAUST SKID & $\begin{array}{l}\text { WILKERSON/DIS471.24 } \\
\text { G-115-4/20MA }\end{array}$ & NA & 22758 ITEM \# 36 & $\begin{array}{l}\text { H-2-829116 SHT } 1 \text { H-14- } \\
100870 \cdot 1,4 \text { ITEM \# } 23\end{array}$ & 6-GENI-135 & GS & NA \\
\hline POR05-VTP-PDI-190 & $\begin{array}{l}\text { TANK S-102 BREATHER FLTR } \\
\text { PRESS DIFF INDICATOR }\end{array}$ & TANK S-102 & OWYER / MODEL 2005 & NA & NA & H-2-73182/73183/73185 & 6-GENI-135 & GS & NA \\
\hline
\end{tabular}




\begin{tabular}{|c|c|c|c|c|c|c|c|c|c|}
\hline EIN & DESCRIPTION & LOCATION & $\begin{array}{l}\text { MANUFACTURER I } \\
\text { MODEL NUMBER }\end{array}$ & SPECIFICATION & $\begin{array}{c}\text { CVI } \\
\text { SUPPLEMENT }\end{array}$ & DRAWING & $\begin{array}{l}\text { MAINTENANCE } \\
\text { PROCEDURE }\end{array}$ & $\begin{array}{l}\text { SAFETY } \\
\text { CLASS }\end{array}$ & $\begin{array}{l}\text { SPARE } \\
\text { PARTS }\end{array}$ \\
\hline POR05.VTP-PDI-191 & $\begin{array}{l}\text { TANK S-103 BREATHER FLTR } \\
\text { PRESS DIFF INDICATOR }\end{array}$ & TANK S-103 & DWYER / MOOEL 2005 & NA & NA & $\mathrm{H}-2-73182 / 73183 / 73185$ & 6.GENI-135 & GS & NA \\
\hline POR05-VTP-FDI-192 & $\begin{array}{l}\text { TANK S-106 BREATHER FLTR } \\
\text { PRESS DIFF INDICATOR } \\
\end{array}$ & TANK S-106 & DWYER /MODEL 2005 & NA & NA & H-2-73182/73183/3185 & 6.GENI-135 & GS & NA \\
\hline POR05-VTP-PDT-170 & $\begin{array}{l}\text { TANK SOURCE I PLENUM INLET } \\
\text { PRESS DIFF XMTR }\end{array}$ & POR05 EXHAUST SKID & $\begin{array}{l}\text { YOKOGAWA / } \# \text { EJA110A } \\
\text { DLS48-92EN/FS1/D1 }\end{array}$ & $\mathrm{NA}$ & 22758 ITEM \# 31 & $\begin{array}{l}\mathrm{H}-2-829116 \text { SHT 2 } \mathrm{H}-2 \cdot \\
73182 / 73183 / 73185\end{array}$ & $6-\mathrm{PCD}-373$ & GS & NA \\
\hline POROS-VTP-PDT-171 & $\begin{array}{l}\text { TANK SOURCE } 1.1 \text { PLENUM INLET } \\
\text { PRESS DIFF XMTR }\end{array}$ & POROS EXHAUST SKID & $\begin{array}{l}\text { YOKOGAWA / \#EJA110A } \\
\text { OLS4B-92EN/FS1/D1 }\end{array}$ & $\mathrm{NA}$ & 22758 ITEM \# 31 & $\begin{array}{l}\mathrm{H}-2 \cdot 829116 \mathrm{SHT} 2 \mathrm{H}-2- \\
73182 \pi 3183 / 73185\end{array}$ & 6.PCD-373 & GS & YES \\
\hline POR05-VTP-PDT-173 & $\begin{array}{l}\text { TANK SOURCE } 2 \text { PLENUM INLET } \\
\text { PRESS DIFF XMTR }\end{array}$ & PORO5 EXHAUST SKID & $\begin{array}{l}\text { YOKOGAWA / \#EJA110A } \\
\text { DLS4B-92EN/FS1/D1 }\end{array}$ & NA & 22758 ITEM \# 31 & H-2-829116 SHT 4 & $6-P C D-373$ & GS & YES \\
\hline POR05-VTP-PDT-177 & $\begin{array}{l}\text { HEAT EXCHANGER } 001 \text { PRESS } \\
\text { DIFF XMTR }\end{array}$ & POR05 EXHAUST SKID & $\begin{array}{l}\text { YOKOGAWA EJA120- } \\
\text { DES48-92EC/FS } 1 / D 1\end{array}$ & $\mathrm{NA}$ & 22758 ITEM \# 31 & $\begin{array}{l}\text { H-2.829116 SHT } 1 \text { H-14- } \\
\text { 100869-1, } 3 \text { ITEM \# } 21\end{array}$ & $6-P C D-373$ & GS & YES \\
\hline POR05-VTP-POT-178 & $\begin{array}{l}\text { PRE FILTER } 001 \text { PRESS DIFF } \\
\text { XMTR }\end{array}$ & PORO5 EXHAUST SKID & $\begin{array}{l}\text { YOKOGAWAIEJA120- } \\
\text { DES4B-92EC/FS1/D1 }\end{array}$ & NA & 22758 ITEM \# 31 & $\begin{array}{l}\mathrm{H}-2-829116 \text { SHT } 1 \mathrm{H}-14- \\
100859-1,3 \text { ITEM } \# 21\end{array}$ & $6-P C D-373$ & GS & YES \\
\hline POR05-VTP-PDT-180 & $\begin{array}{l}\text { 1ST HEPA FILTER 002 PRESS DIFF } \\
\text { XMTR }\end{array}$ & PORO5 EXHAUST SKID & $\begin{array}{l}\text { YOKOGAWA EJA110- } \\
\text { OLS4B-92ECIFS1/D1 OR } \\
\text { EJA110-DLS4B. } \\
\text { 92EN/FS1/D1 }\end{array}$ & NA & 22758 ITEM \# 31 & $\begin{array}{l}\text { H-2-829116 SHT 1 H-14- } \\
100869-1,3 \text { ITEM \# } 22\end{array}$ & $6 . P C D-373$ & ss & YES \\
\hline POR05-VTP.POT-181 & FILTER TRAIN PRESS OIFF XMMTR & PORO5 EXHAUST SKID & $\begin{array}{l}\text { YOKOGAWA EJA110- } \\
\text { DLS4B-92ECIFS1/D1 OR } \\
\text { EJA110-OLSAB- } \\
\text { 92EN/FS1/O1 }\end{array}$ & NA & 22758 ITEM \# 31 & $\begin{array}{l}\text { H-2-829116 SHT 1 H-14- } \\
100869-1,3 \text { ITEM \# } 22\end{array}$ & $6-P C D-373$ & GS & YES \\
\hline POR05-VTP-PDT-182 & 2NO HEPA FILTER 003 XTRM & POR05 EXHAUST SKID & $\begin{array}{l}\text { YOKOGAWA } \\
\text { DLSAB-92ECIFS110. } \\
\text { EJA110-DLS4B. } \\
\text { 92EN/FS1/D1 }\end{array}$ & NA & 22758 ITEM \# 31 & $\begin{array}{l}\text { H-2-829116 SHT } 1 \text { H-14- } \\
100869-1,3 \text { ITEM } \# 22\end{array}$ & $6-P C D-373$ & GS & YES \\
\hline POR5-VTP-PNL-101 & $120 / 240$ SERVICE TO & PORO5 EXHAUST SKIO & SQUARE D / MPZ7S4OF & NA & NA & \begin{tabular}{|l} 
H-14-100868 SHT 1 H-14- \\
100869 SHT 1, 4 ITEM \# \\
1
\end{tabular} & NA & GS & NA \\
\hline POR0S-VTP.PS-101 & $\begin{array}{l}\text { POWER SUPPLY FOR MV-101 AND } \\
\text { MV-102 }\end{array}$ & PORO5 EXHAUST SKID & $\begin{array}{l}\text { POWER ONE / HN24-3.6 } \\
\text { A }\end{array}$ & NA & 22758 ITEM \# 37 & $\begin{array}{l}\mathrm{H}-14-100868 \text { SHT } 6 \text { H-14- } \\
100870 \text { SHT } 2 \text { ITEM \# } 94\end{array}$ & $\mathrm{NA}$ & GS & YES \\
\hline POR05-VTP-PS-301 & GEMS POWER SUPPLY & POROS EXHAUST SKID & $\begin{array}{l}\text { POWER ONE / POWER } \\
\text { SUPPLY, +/-15 VDC } \\
\text { HAA15-0.8A }\end{array}$ & NA & 22758 ITEM \# 47 & $\mid \begin{array}{l}\mid \mathrm{H}-14-100868 \text { SHT } 6 \mathrm{H}-14- \\
100870 \text { SHT } 2 \text { ITEM \# 138 }\end{array}$ & $\overline{N A}$ & GS & YES \\
\hline POR05-VTP-PSH-170 & $\begin{array}{l}\text { TANK SOURCE } 1 \text { PLENUM INLET } \\
\text { PRESS SWITCH HI }\end{array}$ & POR05 EXHAUST SKID & $\begin{array}{l}\text { ACTION INSTRUMENTS } / \\
\text { AP1090-200 }\end{array}$ & NA & 22758 ITEM \# 34 & $\begin{array}{l}\mathrm{H}-2-829116 \text { SHT } 2 \text { H.-14. } \\
100870 \text { SHT } 1,3 \text { \# } 25\end{array}$ & NA & GS & NA \\
\hline POR05-VTP-PSH-171 & $\begin{array}{l}\text { TANK SOURCE } 3 \text { PLENUM INLET } \\
\text { PRESS SWITCH HI }\end{array}$ & POR05 EXHAUST SKID & $\begin{array}{l}\text { ACTION INSTRUMENTS I } \\
\text { AP1090-200 }\end{array}$ & $\mathrm{NA}$ & 22758 ITEM \# 34 & $\begin{array}{l}\mathrm{H}-2-829116 \text { SHT } 1 \mathrm{H}-14 . \\
100870 \text { SHT 1, } 3 \text { ITEM \# } \\
25\end{array}$ & NA & GS & NA \\
\hline POR05-VTP.PSH.173 & $\begin{array}{l}\text { TANK SOURCE } 2 \text { PLENUMM INLET } \\
\text { PRESS SWITCH HI }\end{array}$ & POROS EXHAUST SKID & $\begin{array}{l}\text { ACTION INSTRUMENTS I } \\
\text { AP1090-200 }\end{array}$ & NA & 22758 ITEM \# 34 & $\begin{array}{l}\text { H-2-829116 SHT } 2 \mathrm{H}-14 . \\
100870 \text { SHT 1, } 3 \text { ITEM \# } \\
25\end{array}$ & NA & GS & NA \\
\hline
\end{tabular}




\section{Rev 0}

\begin{tabular}{|c|c|c|c|c|c|c|c|c|c|}
\hline EIN & DESCRIPTION & LOCATION & $\begin{array}{l}\text { MANUFACTURER I } \\
\text { MODEL NUMBER }\end{array}$ & SPECIFICATION & $\begin{array}{c}\text { CVI } \\
\text { SUPPLEMENT }\end{array}$ & DRAWING & $\begin{array}{l}\text { MAINTENANCE } \\
\text { PROCEDURE }\end{array}$ & $\begin{array}{l}\text { SAFETY } \\
\text { CLASS }\end{array}$ & $\begin{array}{l}\text { SPARE } \\
\text { PARTS }\end{array}$ \\
\hline POR05.VTP-RA-301 & $\begin{array}{l}\text { SAMPLE FLOW RADIATION } \\
\text { MONITOR }\end{array}$ & POR05 EXHAUST SKIO & $\begin{array}{l}\text { EBERLINE / AMS4 } \\
\text { OISPLAY UNIT } \\
\text { WIPGMAMSFP } \\
\text { PROGRAM INSTALLED } \\
\text { WITH RELAY OUTPUT, } \\
\text { WITH FAILSAFE } \\
\text { OPERATION }\end{array}$ & NA & 19183 SUPL \# 8 & $\begin{array}{l}\text { H-2-829116 SHT 5/H-14- } \\
100870 \text { SHT 2. } 4 \text { ITEM \# } \\
139\end{array}$ & NA & Ss & YES \\
\hline POR05-VTP-RE-301 & $\begin{array}{l}\text { SAMPLE FLOW RADIATION } \\
\text { ELEMENT }\end{array}$ & POR05 EXHAUST SKID & $\begin{array}{l}\text { EBERLINE / AMS4OPT7- } \\
\text { AMC }\end{array}$ & NA & 19183 SUPL \# 8 & $\begin{array}{l}\text { H-2-829116-5 H-14. } \\
\text { 100916-1, } 16 \text { ITEM \# } 10\end{array}$ & 6.TF-168 & SS & YES \\
\hline POR05-VTP-SP-001 & EXHAUST CONDENSATE ORAIN & PORO5 EXHAUST SKID & SEAL POT ASSEMBLY & $\mathrm{NA}$ & NA & $\begin{array}{l}\text { H-2-829116 SHT } 1 \text { H-14- } \\
100867 \text { SHT } 1 \text { ITEM \# } 8\end{array}$ & $\overline{N A}$ & GS & NA \\
\hline POR05.VTP-SS-102 & EXHAUST CAM SAMPLE PUMPS & POROS EXHAUST SKID & $\begin{array}{l}\text { ALLEN-BRADLEY / 800T- } \\
\text { J2A }\end{array}$ & $\mathrm{NA}$ & NA & $\begin{array}{l}\text { H-2-829116 SHT } 1 \text { H-14- } \\
100870 \text { SHT 2, } 3 \text { ITEM \# } \\
119\end{array}$ & NA & GS & NA \\
\hline POR05-VTP-TE-176 & $\begin{array}{l}\text { HEAT EXCHANGER } 001 \text { INLET } \\
\text { TEMP ELEMENT }\end{array}$ & POR05 EXHAUST SKID & $\begin{array}{l}\text { OMEGA/HWAINB1-2/2- } \\
\text { 1/DH-1-14-J-L3/4-385S- } \\
\text { U161/2-304SS }\end{array}$ & NA & 22758 ITEM \# 17 & $\begin{array}{l}\text { H-2-829116 SHT 1 H-14- } \\
100869 \text { SHT 1, } 3 \text { ITEM \# } \\
24\end{array}$ & NA & $\overline{G S}$ & $\mathrm{NA}$ \\
\hline POR05-VTP-TE-179 & $\begin{array}{l}\text { 1ST HEPA FILTER } 002 \text { INLET TEMP } \\
\text { ELEMENT }\end{array}$ & POR05 EXHAUST SKID & \begin{tabular}{|l} 
OMEGAVHWAINB1-2/2. \\
$1 / D H-1-14-J-L / 3 / 4-385 S-$ \\
U16 1/2-304SS
\end{tabular} & $\overline{N A}$ & 22758 ITEM \# 17 & $\begin{array}{l}\text { H-2-829116 SHT I H-14- } \\
100869 \text { SHT 1, } 3 \text { ITEM \# } \\
24\end{array}$ & NA & GS & NA \\
\hline POR05-VTP-TE-183 & $\begin{array}{l}\text { EXHAUST STACK INLET TEMP } \\
\text { ELEMENT }\end{array}$ & POR05 EXHAUST SKID & $\begin{array}{l}\text { OMEGAHHWA/N81-2/2- } \\
1 / D H-1-4-J-L 3 / 4-385 S-U 4 \\
1 / 2-304 S S\end{array}$ & NA & 22758 ITEM \# 17 & $\begin{array}{l}\text { H-2-829116 SHT } 1 \text { H-14- } \\
100869 \text { SHT } 1,3 \text { ITEM \# } \\
25\end{array}$ & NA & GS & NA \\
\hline POR05-VTP-TI-104 & $\begin{array}{l}\text { HEAT TRACE CABINET } 104 \text { TEMP } \\
\text { INDICATOR }\end{array}$ & POR05 EXHAUST SKID & $\begin{array}{l}\text { MCMASTER-CARR I } \\
4006 K 32\end{array}$ & $\overline{\mathrm{NA}}$ & NA & $\begin{array}{l}\mathrm{H}-2-829116 \text { SHT } 2 \mathrm{H}-14- \\
100870 \text { SHT } 2,3 \text { ITEM \# } \\
60\end{array}$ & NA & $\overline{G S}$ & $\overline{\mathrm{NA}}$ \\
\hline POR05-VTP-Tl-105 & $\begin{array}{l}\text { EXHAUSTER CONTROL PANEL } \\
105 \text { TEMP INDICATOR }\end{array}$ & POR05 EXHAUST SKID & $\begin{array}{l}\text { MCMASTER-CARR I } \\
4006 \mathrm{~K} 32\end{array}$ & NA & NA & $\begin{array}{l}\mathrm{H}-2-829116 \text { SHT } 2 \mathrm{H}-14- \\
100870 \text { SHT } 2,4 \text { ITEM \# } \\
60 \\
\end{array}$ & NA & $\overline{G S}$ & NA \\
\hline POR05.VTP-TI-106 & $\begin{array}{l}\text { INTRINSIC BARRIER CABINET } \\
\text { TEMP INDICATOR }\end{array}$ & POR05 EXHAUST SKID & $\begin{array}{l}\text { MCMASTER-CARR / } \\
4006 \mathrm{~K} 32 \\
\end{array}$ & $\overline{N A}$ & $\overline{N A}$ & $\begin{array}{l}\text { tr-14-100870 SHT 2, } 3 \\
\text { ITEM \# } 60\end{array}$ & NA & & $\overline{\mathrm{NA}}$ \\
\hline POR05-VTR-TI-107 & $\begin{array}{l}\text { ALARM PANEL } 107 \text { TEMP } \\
\text { INDICATOR }\end{array}$ & POR05 EXHAUST SKID & $\begin{array}{l}\text { MCMASTER-CARR / } \\
4006 \mathrm{~K} 32\end{array}$ & NA & NA & $\begin{array}{l}\text { H-2-829116 SHT } 2 \text { H-14- } \\
100870 \text { SHT 2, } 5 \text { ITEM \# } \\
60\end{array}$ & $\overline{N A}$ & GS & $\overline{\mathrm{NA}}$ \\
\hline POR05.VTP.TI-176 & $\begin{array}{l}\text { HEAT EXCHANGER O01 INLET } \\
\text { TEMP INOHCATOR }\end{array}$ & POR05 EXHAUST SKID & $\begin{array}{l}\text { WILKERSON / DIS474-G- } \\
115 \cdot J \cdot \text {.US }\end{array}$ & $\overline{N A}$ & 22758 ITEM \# 35 & $\begin{array}{l}\text { H-2-829116 SHT } 1 \text { H. } 14 \cdot \\
100870 \text { SHT } 1,4 \text { ITEM \# } \\
24\end{array}$ & 6-GENI-135 & GS & $\overline{Y E S}$ \\
\hline POR05-VTP-TI-179 & $\begin{array}{l}\text { 1ST HEPA FILTER } 002 \text { INLET TEMP } \\
\text { INDICATOR }\end{array}$ & POROS EXMAUST SKID & $\begin{array}{l}\text { WILKERSON/DIS 474-G- } \\
\text { 115-J-US }\end{array}$ & NA & 22758 ITEM \# 35 & $\begin{array}{l}\text { H-2-829116 SHT 1 H-14. } \\
100870 \text { SHT } 1,4 \text { ITEM \# } \\
24\end{array}$ & 6-GENI-135 & GS & YES \\
\hline POR05.VTP-TI-183 & $\begin{array}{l}\text { EXHAUST STACK INLET TEMP } \\
\text { INOICATOR }\end{array}$ & POR05 EXHAUST SKID & $\begin{array}{l}\text { WILKERSON / OIS474-G- } \\
115-J-U S\end{array}$ & NA & 22758 ITEM \# 35 & $\begin{array}{l}\text { H-2-829116 SHT } 1 \text { H-14- } \\
100870 \text { SHT } 1 \text {, } 4 \text { ITEM \# } \\
24\end{array}$ & 6-GENI-135 & GS & YES \\
\hline POR05-VTP-TK-001 & GLYCOL EXPANSION TANK & POROS EXHAUST SKID & $\begin{array}{l}\text { HEATER RESERVOIR } \\
\text { ASSEMBLY }\end{array}$ & NA & NA & $\begin{array}{l}\text { H-2-829116 SHT } 1 \text { H-14- } \\
100867 \text { SHT } 1,18 \text { ITEM \# } \\
14\end{array}$ & $\mathrm{NA}$ & GS & NA \\
\hline
\end{tabular}




\begin{tabular}{|c|c|c|c|c|c|c|c|c|c|}
\hline EIN & DESCRIPTION & LOCATION & $\begin{array}{l}\text { MANUFACTURER / } \\
\text { MODEL NUMBER }\end{array}$ & SPECIFICATION & $\begin{array}{c}\text { CV! } \\
\text { SUPPLEMENT }\end{array}$ & DRAWING & $\begin{array}{l}\text { MAINTENANCE } \\
\text { PROCEDURE }\end{array}$ & $\begin{array}{l}\text { SAFETY } \\
\text { CLASS }\end{array}$ & $\begin{array}{l}\text { SPARE } \\
\text { PARTS }\end{array}$ \\
\hline POR0S-VTP-TK-301 & $\begin{array}{l}\text { VACUM PUMP INLET SURGE } \\
\text { SUPPRESSION TANK }\end{array}$ & POR05 EXHAUST SKID & MUFFLER -S/AB612A & NA & NA & $\begin{array}{l}-2-829116 \text { SHT } 1 \text { H-14- } \\
100916 \text { SHT } 1,4 \text { ITEM \# } \\
65\end{array}$ & NA & GS & $\overline{N A}$ \\
\hline POR05-VTP-TS-001 & $\begin{array}{l}\text { HEAT TRACE MASTER CONTROL } \\
\text { SWITCH }\end{array}$ & POROS EXHAUST SKID & $\begin{array}{l}\text { RAYCHEM } \\
\text { CHEMELEXIAMC-1A OR } \\
\text { AMC-18 }\end{array}$ & NA & NA & $\begin{array}{l}\mathrm{H}-2-829116 \text { SHT } 2 / \mathrm{H}-14- \\
100870 \text { SHT } 2,3 \text { ITEM \# } \\
82\end{array}$ & NA & & $\mathrm{NA}$ \\
\hline POR05-VTP-TS-206 & $\begin{array}{l}\text { GLYCOL RECIRCULATING HEATER } \\
001 \text { TEMP SWITCH }\end{array}$ & PORO5 EXHAUST SKID & $\begin{array}{l}\text { CHROMALOX / NWHMT. } \\
\text { 3605E2 }\end{array}$ & $\mathrm{NA}$ & 22758 ITEM \# 29 & $\begin{array}{l}\text { H-2-829116 SHT } 1 \text { H-14- } \\
100867 \text { SHT } 3,18 \text { ITEM \# } \\
136\end{array}$ & NA & GS & NA \\
\hline POR05-VTP-TS-301 & $\begin{array}{l}\text { SAMPLE CABINET } 301 \text { COOLING } \\
\text { FAN TEMP SWITCH }\end{array}$ & POR05 EXHAUST SKID & HOFFMAN/A-TEMNO & NA & NA & $\begin{array}{l}\text { H-2-829116 SHT 5 H-14- } \\
100916 \text { SHT 1, } 4 \text { ITEM \# } \\
19\end{array}$ & $\overline{N A}$ & GS & NA \\
\hline POR05-VTP-TS-302 & $\begin{array}{l}\text { PUMP CABINET } 302 \text { COOLING FAN } \\
\text { TEMP SWITCH }\end{array}$ & POR05 EXHAUST SKID & HOFFMAN/OAH2001A & NA & $\overline{\mathrm{NA}}$ & $\begin{array}{l}\text { H-2-829116 SHT } 5 \text { H-14- } \\
100916 \text { SHT } 1,5 \text { ITEM \# } \\
48\end{array}$ & $\overline{N A}$ & GS & $\overline{N A}$ \\
\hline POR05-VTP-TS-303 & SAMPLE LINE HEAT TRACE & POR05 EXHAUST SKID & RAYCHEM / AMC-18 & $\overline{N A}$ & NA & $\begin{array}{l}\mathrm{H}-2-829116 \text { SHT } 3 \text { H-14. } \\
100916 \text { SHT } 1,4 \text { ITEM \# } 7\end{array}$ & NA & GS & $\overline{N A}$ \\
\hline POR05-VTP.V.101 & PDT 170 MANIFOLD HI SIDE & POR05 EXHAUST SKIO & $\begin{array}{l}\text { D/A MFG CO/GP3TC-T. } \\
\text { LSS-MB }\end{array}$ & NA & 22758 ITEM \# 30 & H-2.829116 SHT 2 & NA & GS & $\overline{N A}$ \\
\hline POR05-VTP-V-102 & POT 170 MANIFOLD LO SIDE & POR05 EXHAUST SKID & $\begin{array}{l}\text { D/A MFG CO / GP3TC-T. } \\
\text { LSS-MB }\end{array}$ & NA & 22758 ITEM \# 30 & H-2-829116 SHT 2 & $\overline{N A}$ & GS & NA \\
\hline POR05-VTP-V-103 & $\begin{array}{l}\text { PDT } 170 \text { MANIFOLD EQUALIZING } \\
\text { VALVE }\end{array}$ & POR05 EXHAUST SKID & $\begin{array}{l}\text { D/A MFG CO / GP3TC-T- } \\
\text { LSS-MB }\end{array}$ & NA & 22758 ITEM \# 30 & $\begin{array}{l}\text { l-2-829116 SHT } 2 \mathrm{H}-14- \\
100867 \text { SHT 1, } 7 \text { ITEM \# } \\
40\end{array}$ & $\overline{\mathrm{NA}}$ & GS & NA \\
\hline POR05-VTP-V-104 & PDT 171 MANIFOLD HI SIDE & POR05 EXHAUST SKID & $\begin{array}{l}\text { D/A MFG CO / GP3TC-T- } \\
\text { LSS-MB }\end{array}$ & NA & 22758 ITEM \# 30 & H-2-829116 SHT 4 & NA & GS & $\overline{N A}$ \\
\hline POR05.VTP.V-105 & PDT 171 MANIFOLD LO SIDE & POROS EXHAUST SKID & $\begin{array}{l}\text { D/A MFG CO / GP3TC-T- } \\
\text { LSS-MB }\end{array}$ & NA & 22758 ITEM \# 30 & $\mathrm{H}-2-829116 \mathrm{SHT} 4$ & NA & GS & $\overline{N A}$ \\
\hline POR05-VTP-V-106 & $\begin{array}{l}\text { POT } 171 \text { MANIFOLO EQUJALIZING } \\
\text { VALVE }\end{array}$ & POR05 EXHAUST SKID & $\begin{array}{l}\text { DIA MFG CO/GP3TC-Y. } \\
\text { LSS-MB }\end{array}$ & NA & 22758 ITEM \# 30 & $\begin{array}{l}\text { H-2.829116 SHT } 4 \mathrm{H}-14- \\
100867 \text { SHT 1, } 7 \text { ITEM \# } \\
40\end{array}$ & NA & GS & NA \\
\hline POR0S-VTP.V-111 & FT $172 \mathrm{HI}$ SIOE FIRST & POR05 EXHAUST SKID & $\begin{array}{l}\text { VERIS VERABAR / } \\
\text { MODELS V100, V110 }\end{array}$ & $\overline{N A}$ & $\begin{array}{c}22758 \text { ITEM \# } 40, \\
41\end{array}$ & $\begin{array}{l}\text { H-2-829116 SHT 4 H-14- } \\
100867 \text { SHT 1, } 9 \text { ITEM \# } \\
18\end{array}$ & NA & GS & $\overline{\mathrm{NA}}$ \\
\hline POR05-VTP-V-112 & Fr 172 LO SIDE FIRST & POR05 EXHAUST SKID & $\begin{array}{l}\text { VERIS VERABAR I } \\
\text { MODELS } V 100, V 110\end{array}$ & NA & \begin{tabular}{|c|}
22758 IrEM \# 40, \\
41 \\
\end{tabular} & $\begin{array}{l}\mathrm{H}-2-829116 \mathrm{SHT} 4 \mathrm{H}-14- \\
100867 \mathrm{SHT} 1,9 \text { ITEM \# } \\
18\end{array}$ & $\overline{N A}$ & GS & NA \\
\hline POR05-VTP-V-113 & FT 172 HI SIDE CAL & POR05 EXHAUST SKID & $\begin{array}{l}\text { D/A MFG CO/GP3TC.T. } \\
\text { LSS-MB }\end{array}$ & $\overline{N A}$ & 22758 ITEM \# 30 & H-2-829116 SHT 4 & $\overline{\mathrm{NA}}$ & GS & $\overline{\mathrm{NA}}$ \\
\hline POR05.VTP.V-114 & FT 172 LO SIDE CAL & POR05 EXHAUST SKID & $\begin{array}{l}\text { D/A MFG CO/GP3TC.Y. } \\
\text { LSS-MB }\end{array}$ & NA & 22758 ITEM \# 30 & $\mathrm{H} \cdot 2.829116 \mathrm{SHT} 4$ & $\overline{N A}$ & $\overline{G S}$ & NA \\
\hline POR05-VTP-V-115 & $\begin{array}{l}\text { FT } 172 \text { MANIFOLD HI SIDE } \\
\text { SECOND }\end{array}$ & POR05 EXHAUST SKIO & $\begin{array}{l}\text { D/A MFG CO/GP3TC-Y- } \\
\text { LSS-MB }\end{array}$ & NA & $2275817 E M \# 30$ & H-2.829116 SHT 4 & NA & GS & NA \\
\hline POR05-VTP-V-116 & $\begin{array}{l}\text { FT } 172 \text { MANIFOLD LO SIDE } \\
\text { SECOND }\end{array}$ & POR05 EXHAUST SKID & $\begin{array}{l}\text { D/A MFG CO / GP3TC-T. } \\
\text { LSS-MB }\end{array}$ & $\overline{N A}$ & 22758 ITEM \# 30 & H-2.829116 SHT 4 & $\overline{N A}$ & GS & NA \\
\hline
\end{tabular}




\begin{tabular}{|c|c|c|c|c|c|c|c|c|c|}
\hline EIN & DESCRIPTION & LOCATION & $\begin{array}{l}\text { MANUFACTURER I } \\
\text { MODEL NUMBER }\end{array}$ & SPECIFICATION & $\begin{array}{c}\text { CV! } \\
\text { SUPPLEMENT }\end{array}$ & DRAWING & $\begin{array}{l}\text { MAINTENANCE } \\
\text { PROCEDURE }\end{array}$ & $\begin{array}{l}\text { SAFETY } \\
\text { CLASS }\end{array}$ & $\begin{array}{l}\text { SPARE } \\
\text { PARTS }\end{array}$ \\
\hline POR0S-VTP-V-117 & $\begin{array}{l}\text { FT } 172 \text { MANIFOLD EQUALIZING } \\
\text { VALVE }\end{array}$ & PORO5 EXHAUST SKID & $\begin{array}{l}\text { D/A MFG CO/GP3TC-T. } \\
\text { LSS-MB }\end{array}$ & NA & 22758 ITEM \# 30 & $\begin{array}{l}\text { H-2-829116 SHT 4 H-14- } \\
100867 \text { SHT 1, } 7 \text { ITEM \# } \\
40\end{array}$ & NA & GS & NA \\
\hline POR05-VTP-V-118 & PDT-173 MANIFOLO HI SIDE & POROS EXHAUST SKID & $\begin{array}{l}\text { D/A MFG CO/GP3TC-T- } \\
\text { LSS-MB }\end{array}$ & NA & 22758 ITEM \# 30 & $\mathrm{H}-2-829116$ SHT 2 & NA & GS & NA \\
\hline POR05-VTP-V-119 & PDT-173 MANIFOLO LO SIDE & POROS EXHAUST SKID & $\begin{array}{l}\text { D/A MFG CO/GP3TC-T- } \\
\text { LSS-MB }\end{array}$ & NA & 22758 ITEM $\# 30$ & H-2.829116 SHT 2 & NA & GS & NA \\
\hline POR05-VTP-V-120 & $\begin{array}{l}\text { PDT-173 MANIFOLD EQUALIZING } \\
\text { VALVE }\end{array}$ & POROS EXHAUST SKID & $\begin{array}{l}\text { D/A MFG CO/GP3TC-T- } \\
\text { LSS-MB }\end{array}$ & NA & 22758 ITEM \# 30 & $\begin{array}{l}\text { H-2-829116 SHT } 2 \mathrm{H}-14 . \\
100867 \text { SHT } 1,7 \text { ITEM \# } \\
40\end{array}$ & NA & GS & NA \\
\hline POR05-VTP.V.121 & FT $174 \mathrm{HI}$ SIDE FIRST & POR05 EXHAUST SKIO & $\begin{array}{l}\text { VERIS VERABAR/ } \\
\text { MODELS V } 100, V 110\end{array}$ & NA & $\begin{array}{c}22758 \text { ITEM \# 40, } \\
41\end{array}$ & $\begin{array}{l}\text { H-2-829116 SHT } 2 \text { H-14- } \\
100870 \text { SHT 1, } 9 \text { ITEM \# } \\
18\end{array}$ & NA & GS & $\overline{\mathrm{NA}}$ \\
\hline POR05-VTP-V-122 & FT 174 LO SIDE FIRST & POR05 EXHAUST SKID & $\begin{array}{l}\text { VERIS VERABAR I } \\
\text { MODELS V100, V110 }\end{array}$ & NA & $\begin{array}{c}22758 \text { ITEM \# } 40, \\
41\end{array}$ & $\begin{array}{l}\text { H-2-829116 SHT } 2 \text { H-14- } \\
100870 \text { SHT 1, } 9 \text { ITEM \# } \\
18\end{array}$ & NA & GS & NA \\
\hline POR05-VIP-V-123 & FT $174 \mathrm{HI}$ SIDE CAL & POROS EXHAUST SKID & $\begin{array}{l}\text { D/A MFG CO /GP3TC-T- } \\
\text { LSS-MB }\end{array}$ & NA & 22758 ITEM \# 30 & $\mathrm{H}-2-829116$ SHT 2 & NA & GS & NA \\
\hline POR05-VTP.V-124 & FT 174 LO SIOE CAL & POR05 EXHAUST SKID & $\begin{array}{l}\text { D/A MFG CO / GP3TC-T. } \\
\text { LSS-MB }\end{array}$ & NA & 22758 ITEM \# 30 & $\mathrm{H}-2-829116$ SHT 2 & NA & GS & NA \\
\hline POR05-VTP-V-125 & $\begin{array}{l}\text { FT } 174 \text { MANIFOLD HI SIDE } \\
\text { SECOND }\end{array}$ & POR05 EXHAUST SKID & $\begin{array}{l}\text { D/A MFG CO/GP3TC-T- } \\
\text { LSS.MB }\end{array}$ & NA & 22758 ITEM \# 30 & H-2-829116 SHT 2 & NA & GS & $\mathrm{NA}$ \\
\hline POR05-VTP-V-126 & $\begin{array}{l}\text { FT } 174 \text { MANIFOLD LO SIDE } \\
\text { SECOND }\end{array}$ & POROS EXHAUST SKID & $\begin{array}{l}\text { D/A MFG CO / GP3TC-T- } \\
\text { LSS-MB }\end{array}$ & $\mathrm{NA}$ & 22758 ITEM $\# 30$ & $\mathrm{H}-2.829116$ SHT 2 & NA & GS & NA \\
\hline POR0S.VTP.V.127 & $\begin{array}{l}\text { FT 174 MANIFOLD EQUALIZING } \\
\text { VALVE }\end{array}$ & PORO5 EXHAUST SKID & $\begin{array}{l}\text { D/A MFG CO / GP3TC-T. } \\
\text { LSS-MB }\end{array}$ & NA & 22758 ITEM \# 30 & $\begin{array}{l}\mathrm{H}-2-829116 \text { SHT } 2 \mathrm{H}-14 . \\
100867 \text { SHT } 1,7 \text { ltem } \sharp \\
40\end{array}$ & $\mathrm{NA}$ & GS & $\mathrm{NA}$ \\
\hline POR05-VTP.V.135 & $\begin{array}{l}\text { 12" PRI VENT EXHAUST TRAIN } \\
\text { INLET }\end{array}$ & POR05 EXHAUST SKID & $\begin{array}{l}\text { KEYSTONE / FIG } 360 \\
\text { TRIM CODE } 173\end{array}$ & NA & 22758 ITEM \# 9 & $\begin{array}{l}\mathrm{H}-2-829116 \mathrm{SHT} 1 \mathrm{H}-14 . \\
100867 \mathrm{SHT} 1,6 \text { Item } \# 21\end{array}$ & NA & SS & NA \\
\hline POR05-VTP.V.136 & $\begin{array}{l}\text { 6" PRI VENT EXHAUST TRAIN } \\
\text { OUTLET }\end{array}$ & POR05 EXHAUST SKID & $\begin{array}{l}\text { KEYSTONE / FIG } 360 \\
\text { TRIM CODE } 173\end{array}$ & NA & 22758 ITEM \# 9 & $\begin{array}{l}\text { H-2-829116 SHT } 1 \text { H-14- } \\
100867 \text { SHT } 1,6 \text {, Item \# } \\
16\end{array}$ & NA & ss & NA \\
\hline POR05-VTP-V-137 & PDT 177 MANIFOLD HI SIOE & POROS EXHAUST SKID & $\begin{array}{l}\text { D/A MFG CO / GP3TC-T- } \\
\text { LSS-MB }\end{array}$ & NA & 22758 ITEM \# 30 & $\mathrm{H}-2-829116$ SHT 1 & NA & GS & NA \\
\hline POR05-VTP-V-138 & PDT 177 MANIFOLO LO SIDE & POROS EXHAUST SKID & $\begin{array}{l}\text { D/A MFG CO / GP3TC-T- } \\
\text { LSS-MB }\end{array}$ & NA & 22758 ITEM \#30 & $\mathrm{H}-2-829116 \mathrm{SHT} 1$ & NA & GS & NA \\
\hline POR05-VTP.V.139 & $\begin{array}{l}\text { PDT } 177 \text { MANIFOLO EQUALIZING } \\
\text { VALVE }\end{array}$ & POR05 EXHAUST SKIO & $\begin{array}{l}\text { DIA MFG CO / GP3TC.T. } \\
\text { LSS-MB }\end{array}$ & NA & 22758 ITEM \# 30 & $\begin{array}{l}\text { H-2-829116 SHT } 1 \text { H-14. } \\
100870 \text { SHT } 1,7 \text { ltem \# } \\
40\end{array}$ & NA & GS & NA \\
\hline POR05.VTP-V-140 & POT 178 MANIFOLD HI SIDE & POR05 EXHAUST SKID & $\begin{array}{l}\text { O/A MFG CO/GP3TC-T- } \\
\text { LSS-MB }\end{array}$ & NA & 22758 ITEM $\# 30$ & $\mathrm{H}-2-829116$ SHT 1 & NA & GS & NA \\
\hline POR05-VTP-V-141 & PDT 178 MANIFOLO LO SIDE & POR05 EXHAUST SKIO & NA. & NA & 22758 ITEM $\# 30$ & H-2-829116 SHT 1 & NA & GS & NA \\
\hline POR05-VTP-V-142 & $\begin{array}{l}\text { PDT } 178 \text { MANIFOLO EQUALIZING } \\
\text { VALVE }\end{array}$ & PORO5 EXHAUST SKID & $\begin{array}{l}\text { DIA MFG CO/GP3TC-T- } \\
\text { LSS-MB }\end{array}$ & NA & 22758 ITEM \# 30 & $\begin{array}{l}\text { H-2-829116 SHT } 1 \text { H-14- } \\
100870 \text { SHT } 1,7 \text { ltem } \# \\
40\end{array}$ & $\mathrm{NA}$ & GS & $\mathrm{NA}$ \\
\hline POR05-VTP-V-143 & PDT 180 MANIFOLD HI SIDE & POR05 EXHAUST SKIO & $\begin{array}{l}\text { D/A MFG CO/GP3TC-T- } \\
\text { LSS-MB }\end{array}$ & NA & 22758 ITEM \# 30 & $\mathrm{H}-2-829116 \mathrm{SHT} 1$ & NA & GS & NA \\
\hline
\end{tabular}




\begin{tabular}{|c|c|c|c|c|c|c|c|c|c|}
\hline EIN & DESCRIPTION & LOCATION & $\begin{array}{l}\text { MANUFACTURER / } \\
\text { MODEL NUMBER }\end{array}$ & SPECIFICATION & $\begin{array}{c}\text { CVI } \\
\text { SUPPLEMENT }\end{array}$ & DRAWING & $\begin{array}{l}\text { MAINTENANCE } \\
\text { PROCEDURE }\end{array}$ & $\begin{array}{l}\text { SAFETY } \\
\text { CLASS }\end{array}$ & $\begin{array}{l}\text { SPARE } \\
\text { PARTS }\end{array}$ \\
\hline POR0S.VTP-V-144 & PDT 180 MANIF̈OLD LO SIDE & POR05 EXHAUST SKID & $\begin{array}{l}\text { D/A MFG CO / GP3TC.T. } \\
\text { LSS-MB }\end{array}$ & NA & 22758 ITEM $\# 30$ & प्र-2-829116 SHT 1 & NA & GS & NA \\
\hline POR05.VTP-V-145 & $\begin{array}{l}\text { POT } 180 \text { MANIFOLD EQUALIZING } \\
\text { VALVE }\end{array}$ & POR05 EXHAUST SKID & $\begin{array}{l}\text { D/A MFG CO / GP3TC-T. } \\
\text { LSS-MB }\end{array}$ & NA & 22758 ITEM \# 30 & $\begin{array}{l}\mathrm{H}-2-829116 \text { SHT } 1 \mathrm{H}-14 . \\
100870 \text { SHT } 1,7 \text { Item \# } \\
40\end{array}$ & NA & GS & $\mathrm{NA}$ \\
\hline POR05-VTP-V-146 & PDT 181 MANIFOLD HI SIDE & POR05 EXHAUST SKID & $\begin{array}{l}\text { D/A MFG CO /GP3TC-T- } \\
\text { LSS-M8 }\end{array}$ & NA & 22758 ITEM \# 30 & H-2.829116 SHT 1 & NA & GS & NA \\
\hline POR05-VTP-V-147 & PDT 181 MANIFOLO LO SIDE & PORO5 EXHAUST SKID & $\begin{array}{l}\text { D/A MFG CO/GP3TC-T- } \\
\text { LSS-MB }\end{array}$ & $\overline{\mathrm{NA}}$ & 22758 ITEM \# 30 & H-2-829116 SHT 1 & NA & GS & NA \\
\hline POR05-VTP-V-148 & $\begin{array}{l}\text { PDT } 181 \text { MANIFOLO EQUALIZING } \\
\text { VALVE }\end{array}$ & POR05 EXHAUST SKID & $\begin{array}{l}\text { O/A MFG CO / GP3TC-T- } \\
\text { LSS-MB }\end{array}$ & $\overline{N A}$ & 22758 ITEM \# 30 & $\begin{array}{l}\text { H-2-829116 SHT } 1 \text { H-14- } \\
100870 \text { SHT } 1,7 \text { ltem \# } \\
40\end{array}$ & NA & GS & NA \\
\hline POR05-VTP-V-149 & PDT 182 MANIFOLD SIOE & POR05 EXHAUST SKID & $\begin{array}{l}\text { D/A MFG CO/GP3TC-T. } \\
\text { LSS-MB }\end{array}$ & NA & 22758 ITEM $\# 30$ & H-2-829116 SHT 1 & NA & GS & NA \\
\hline POROS-VTP-V-150 & PDT 182 MANIFOLD LO SIOE & POR05 EXHAUST SKID & $\begin{array}{l}\text { D/A MFG CO / GP3TC-T- } \\
\text { LSS-MB }\end{array}$ & NA & 22758 ITEM $\# 30$ & H-2.829116 SHT 1 & NA & GS & NA \\
\hline POR05-VTP-V-151 & $\begin{array}{l}\text { POT } 182 \text { MANIFOLD EQUALIZING } \\
\text { VALVE }\end{array}$ & POROS EXHAUST SKID & $\begin{array}{l}\text { D/A MFG CO/GP3TC-T- } \\
\text { LSS-MB }\end{array}$ & NA & 22758 ITEM \# 30 & $\begin{array}{l}\text { H-2.829116 SHT 1 H-14- } \\
100870 \text { SHT } 1,7 \text { item \# } \\
40\end{array}$ & NA & GS & NA \\
\hline POR0S-VTP-V-153 & FE 184 HISIDE VALVE & POR05 EXHAUST SKIO & $\begin{array}{l}\text { VERIS VERABAR I } \\
\text { MODELS V100, V110 }\end{array}$ & $\mathrm{NA}$ & $\begin{array}{c}22758 \text { ITEM \# } 40, \\
41\end{array}$ & $\begin{array}{l}\text { H-2.829116 SHT } 1 \text { H-14- } \\
100870 \text { SHT } 1,9 \text { Item \# } \\
18\end{array}$ & $\mathrm{NA}$ & GS & $\mathrm{NA}$ \\
\hline POR05.VTP-V-154 & FE 184 LO SIDE VALVE & POR05 EXHAUST SKID & $\begin{array}{l}\text { VERIS VERABAR I } \\
\text { MODELS V100, V110 }\end{array}$ & $\mathrm{NA}$ & $\begin{array}{c}22758 \text { ITEM \# 40, } \\
41\end{array}$ & $\begin{array}{l}\text { H-2-829116 SHT } 1 \mathrm{H}-14- \\
100870 \text { SHT } 1,9 \text { tem \# } \\
18\end{array}$ & NA & GS & NA \\
\hline POR05-VTP-V-155 & FT 184 MANIFOLD HI SIOE VALVE & POR05 EXHAUST SKID & $\begin{array}{l}\text { D/A MFG CO/GP3TC-T- } \\
\text { LSS.MB }\end{array}$ & $\mathrm{NA}$ & 22758 ITEM $\# 30$ & H.2.829116 SHT 1 & $\overline{N A}$ & GS & NA \\
\hline POR05.VIP-V-156 & FT 184 MANIFOLD LO SIDE VALVE & POROS EXHAUST SKID & $\begin{array}{l}\text { D/A MFG CO / GP3TC-T- } \\
\text { LSS-MB }\end{array}$ & NA & 22758 ITEM \# 30 & H-2-829116 SHT 1 & NA & GS & NA \\
\hline POR05-VTP-V-157 & $\begin{array}{l}\text { FT } 184 \text { MANIFOLD EQUALIZING } \\
\text { VALVE }\end{array}$ & POR05 EXHAUST SKID & $\begin{array}{l}\text { D/A MFG CO / GP3TC-T. } \\
\text { LSS-MB }\end{array}$ & NA & 22758 ITEM \# 30 & $\begin{array}{l}\text { H-2-829116 SHT 1 H-14- } \\
100870 \text { SHT 1, } 7 \text { Item \# } \\
40\end{array}$ & NA & GS & NA \\
\hline POR05-VTP-V-158 & VENT TRAIN EXHAUST FAN VALVE & PORO5 EXHAUST SKIO & $\begin{array}{l}\text { WHITEY / SS-65TF16. } \\
\text { LLK, SS-65TW16P40-LLKK }\end{array}$ & NA & NA & \begin{tabular}{|l|}
$\mathrm{H}-2-829116$ SHT $1 \mathrm{H}-14-$ \\
100867 SHTT $2,3,7$ Item \# \\
$96 \% 155$
\end{tabular} & $\mathrm{NA}$ & GS & NA \\
\hline POR05-VTP-V. 160 & SEAL POT 001 DRAIN VALVE & POR05 EXHAUST SKID & $\begin{array}{l}\text { WHITEY / SS-63TW8P40- } \\
\text { LLK }\end{array}$ & NA & NA & $\begin{array}{l}\text { H-2-829116 SHT } 1 \text { H-14 } \\
100867 \text { SHT 4, } 16 \text { Item \# } \\
160\end{array}$ & NA & GS & NA \\
\hline POR05-VTP-V-161 & SEAL POT 001 OVERFLOW VALVE & POR05 EXHAUST SKID & $\begin{array}{l}\text { WHITEYISS-65TW16P40- } \\
\text { LLK }\end{array}$ & NA & NA & $\begin{array}{l}\mathrm{H}-2-829116 \mathrm{SHT} \text { i H-14- } \\
100867 \mathrm{SHT} 3,7,16 \text { Item } \\
\# 155\end{array}$ & NA & GS & NA \\
\hline POR05.VTP.V-162 & SEAL POT 001 VENT VALVE & PORO5 EXHAULST SKID & $\begin{array}{l}\text { WHITEY / SS-65TF16- } \\
\text { LLLK, SS-65TW16P40-LLK } \\
\text { ITEM \# } 96 \# 155\end{array}$ & NA & NA & $\begin{array}{l}\mathrm{H}-2-829116 \text { SHT } 1 \mathrm{H}-14- \\
100867 \mathrm{SHT} 2,3,7 \text { Item \# } \\
96 \text { \# } 155\end{array}$ & $\overline{N A}$ & GS & NA \\
\hline POR05-VTP-V.163 & $\begin{array}{l}\text { SEAL POT PUMP } 004 \text { RETURN } \\
\text { VALVE }\end{array}$ & POR05 EXHAUST SKID & $\begin{array}{l}\text { WHITEY / SS-63TW8P40- } \\
\text { LLK OR SS-63TW8P40 } \\
\text { WISS-51K-63LLK-OG } \\
\text { HANDLE KIT }\end{array}$ & NA & NA & $\begin{array}{l}\text { H-2.829116 SHT } 1 \text { H-14- } \\
100867 \text { SHT 5, } 22 \text { Item \# } \\
245\end{array}$ & NA & GS & $\mathrm{NA}$ \\
\hline
\end{tabular}




\begin{tabular}{|c|c|c|c|c|c|c|c|c|c|}
\hline EIN & DESCRIPTION & LOCATION & $\begin{array}{l}\text { MANUFACTURER I } \\
\text { MODEL NUMBER }\end{array}$ & SPECIFICATION & $\begin{array}{c}\text { CVI } \\
\text { SUPPLEMENT }\end{array}$ & DRAWING & $\begin{array}{l}\text { MAINTENANCE } \\
\text { PROCEDURE }\end{array}$ & $\begin{array}{l}\text { SAFETY } \\
\text { CLASS }\end{array}$ & $\begin{array}{l}\text { SPARE } \\
\text { PARTS }\end{array}$ \\
\hline POR05-VTP.V-164 & $\begin{array}{l}\text { SEAL POT PUMP } 004 \text { SUCTION } \\
\text { VALVE }\end{array}$ & PORO5 EXHAUST SKID & $\begin{array}{l}\text { WHITEY / SS-63TW8P40- } \\
\text { LLK OR SS-63TW8P40 } \\
\text { WISS-51K-63LLK-OG } \\
\text { HANDLE KIT }\end{array}$ & $\mathrm{NA}$ & NA & $\begin{array}{l}\text { H.2-829116 SHT } 1 \text { H-14- } \\
100867 \text { SHT } 5,22 \text { ltem \# } \\
245\end{array}$ & $\overline{N A}$ & GS & $\mathrm{NA}$ \\
\hline POR05-VTP-V-165 & $\begin{array}{l}\text { SEAL POT PUMP ENCLOSURE } \\
\text { INLET VALVE }\end{array}$ & POR05 EXHAUST SKID & $\begin{array}{l}\text { WHITEY / SS-63TW8P40- } \\
\text { LLK OR SS-63TW8P40 } \\
\text { W/SS-51K-63LLK-OG } \\
\text { HANDLE KIT }\end{array}$ & NA & NA & $\begin{array}{l}\mathrm{H}-2 \cdot 829116 \text { SHT } 1 \text { H-14. } \\
100867 \text { SHT S, } 22 \text { Item \# } \\
245\end{array}$ & $\mathrm{NA}$ & GS & NA \\
\hline POR05-VTP-V-166 & $\begin{array}{l}\text { SEAL POT PUMP } 004 \text { DISCH TO } \\
\text { TANK VALVE }\end{array}$ & POR05 EXHAUST SKID & $\begin{array}{l}\text { WHITEY / SS-63TW8P40- } \\
\text { LLK OR SS-63TW8P40 } \\
\text { WISS-51K-63LLK-OG } \\
\text { HANDLE KIT }\end{array}$ & NA & $\overline{\mathrm{NA}}$ & $\begin{array}{l}\text { H-2-829116 SHT } 1 \text { H-14. } \\
100867 \text { SHT 5, } 22 \text { ltem \# } \\
245\end{array}$ & NA & $\overline{G S}$ & $\overline{N A}$ \\
\hline PORO5-VTP-V-180 & $\begin{array}{l}\text { EXHAUSTER CONDENSATE } \\
\text { DRAIN ISO VALVE }\end{array}$ & TANK S.102 & $\begin{array}{l}\text { WHITEY/ SS-65TW16P40 } \\
\mathrm{JL}\end{array}$ & NA & NA & $\mathrm{H}-2-73183$ & NA & GS & NA \\
\hline POR05-VIP.V.181 & $\begin{array}{l}\text { EXHAUSTER INLET ISOLATION } \\
\text { FROM TANK S-102 }\end{array}$ & TANK S.102 & $\begin{array}{l}\text { KEYSTONE / 8" LUG } \\
\text { TYPE B'FLY VALVE ANSI } \\
\text { CLASS } 150 \text { FULL LUG } \\
\text { F362-173 W/GEAR } \\
\text { OPERATOR }\end{array}$ & NA & NA & $\mathrm{H}-2-73183$ & $\overline{N A}$ & GS & $\overline{\mathrm{NA}}$ \\
\hline POR05-VTP.V-182 & $\begin{array}{l}\text { TANK S.102 ISOLATION TO } \\
\text { PORTABLE EXHAUSTER }\end{array}$ & TANK S-102 & \begin{tabular}{|l|} 
KEYSTONE $/ 8 "$ LUG \\
TYPE B'FLY VALVE ANSI \\
CLASS 150 FULL LUG \\
F362-173 W/GEAR \\
OPERATOR
\end{tabular} & NA & NA & H.2.73182 & NA & GS & $\mathrm{NA}$ \\
\hline POR05-VTP-V-183 & $\begin{array}{l}\text { TANK S-102 BREATHER FILTER } \\
\text { ISOLATION VALVE }\end{array}$ & TANK S-102 & $\begin{array}{l}\text { KEYSTONE / } 12 " \text { LUG K- } \\
\text { LOK BUTTERFLY VALVE } \\
\text { ANSI CLASS } 150 \text { FULL } \\
\text { LUG } 12 \text { F362-173 }\end{array}$ & NA & NA & $\mathrm{H}-2-73182 \pi 3183 / 73185$ & $\overline{\mathrm{NA}}$ & GS & NA \\
\hline POR05-VTP-V-184 & $\begin{array}{l}\text { TANK S-102 BREATHER FILTER } \\
\text { CONDENSATE DRAIN VALVE }\end{array}$ & TANK S-102 & SWAGELOK / \#SS63TS8 & $\overline{\mathrm{NA}}$ & NA & $4 \cdot 2-73182 / 73183 / 73185$ & NA & GS & NA \\
\hline POR05-VTP.V-185 & $\begin{array}{l}\text { EXHAUST INLET ISOLATION FROM } \\
\text { TANK S-103 }\end{array}$ & TANK S-103 & \begin{tabular}{|l|} 
KEYSTONE / 8" LUG \\
TYPE B'FLY VALVE ANSI \\
CLASS 150 FULLL LUG \\
F362-173 W/GEAR \\
OPERATOR
\end{tabular} & $\overline{\mathrm{NA}}$ & NA & $\mathrm{H}-2.73182 / 73183733185$ & $\overline{N A}$ & GS & NA \\
\hline POR05-VTP-V-186 & $\begin{array}{l}\text { TANK S-103 ISOLATION TO } \\
\text { PORTABLE EXHAUSTER }\end{array}$ & TANK S-103 & \begin{tabular}{|l|} 
KEYSTONE / 8" LUG \\
TYPE B'FLY VALVE ANSI \\
CLASS 150 FULL LUG \\
F362-173 W/GEAR \\
OPERATOR
\end{tabular} & $\overline{N A}$ & NA & $\mathrm{H}-2.73183$ & $\overline{\mathrm{NA}}$ & GS & $\overline{N A}$ \\
\hline POR05-VTP-V-187 & $\begin{array}{l}\text { TANKS S-103 BREATHER FILTER } \\
\text { ISOLATION VALVE }\end{array}$ & TANK S-103 & $\begin{array}{l}\text { KEYSTONE / } 12 \text { "LUG K- } \\
\text { LOK BUTTERFLY VALVE } \\
\text { ANSI CLASS } 150 \text { FULL } \\
\text { LUG } 12 \text {. } 362.173\end{array}$ & NA & $\overline{N A}$ & $\mathrm{H} \cdot 2.73182 / 7318373185$ & NA & GS & NA \\
\hline POR05-VTP-V-188 & $\begin{array}{l}\text { TANKS S-103 BREATHER FILTER } \\
\text { CONDENSATE DRAIN VALVE }\end{array}$ & TANK S-103 & SWAGELOK / \#SS63TS8 & $\overline{N A}$ & $\overline{\mathrm{NA}}$ & $H-2-73182 / 73183 / 73185$ & NA & GS & NA \\
\hline
\end{tabular}




\begin{tabular}{|c|c|c|c|c|c|c|c|c|c|}
\hline EIN & DESCRIPTION & LOCATION & $\begin{array}{l}\text { MANUFACTURER/' } \\
\text { MODEL. NUMBER }\end{array}$ & SPECIFICATION & $\begin{array}{c}\text { CVI } \\
\text { SUPPLEMENT }\end{array}$ & DRAWING & $\begin{array}{l}\text { MAINTENANCE } \\
\text { PROCEDURE }\end{array}$ & $\begin{array}{l}\text { SAFETY } \\
\text { CLASS }\end{array}$ & $\begin{array}{l}\text { SPARE } \\
\text { PARTS }\end{array}$ \\
\hline POR05-VTP-V-189 & $\begin{array}{l}\text { EXHAUST INLET ISOLATION FROM } \\
\text { TANK S-016 }\end{array}$ & TANK S.106 & \begin{tabular}{|l|} 
KEYSTONE / 8" LUG \\
TYPE B'FLY VALVE ANSI \\
CLASS 150 FULLL LUG \\
F362-173 W/GEAR \\
OPERATOR
\end{tabular} & NA & NA & $\mathrm{H}-2.73182 / 73183 / 73185$ & NA & GS & NA \\
\hline POR05-VTP.V-190 & $\begin{array}{l}\text { TANK S-106 ISOLATION TO } \\
\text { PORTABLE EXHAUSTER }\end{array}$ & TANK S-106 & $\begin{array}{l}\text { KEYSTONE / } 8 " \text { "LUG } \\
\text { TYPE B'FLY VALVE ANSI } \\
\text { CLASS } 150 \text { FULL LUG } \\
\text { F362-173 W/GEAR } \\
\text { OPERATOR }\end{array}$ & NA & NA & $\mathrm{H} \cdot 2 \cdot 73185$ & $\overline{N A}$ & GS & NA \\
\hline POR05-VTP-V-191 & $\begin{array}{l}\text { TANK S-106 BREATHER FILTER } \\
\text { ISOLATION VALVE }\end{array}$ & TANK S-106 & $\begin{array}{l}\text { KEYSTONE / 12" LUG K- } \\
\text { LOK BUTTERFLY VALVE } \\
\text { ANSI CLASS } 150 \text { FULL } \\
\text { LUG } 12 \text { F362-173 }\end{array}$ & $\overline{\mathrm{NA}}$ & $\overline{\mathrm{NA}}$ & $\mathrm{H}-2.73182 / 73183773185$ & NA & GS & NA \\
\hline POR4-VTP-V-192 & $\begin{array}{l}\text { TANKS S-106 BREATHER FILTER } \\
\text { CONOENSATE DRAIN VALVE }\end{array}$ & TANK $\$ .106$ & SWAGELOK/\#SS63TS8 & $\overline{N A}$ & $\overline{N A}$ & $\mathrm{H}-2.73182 / 73183 / 73185$ & NA & GS & NA \\
\hline POR05.VTP.V.201 & $\begin{array}{l}\text { GLYCOL PUMP 001 SUCTION } \\
\text { VALVE }\end{array}$ & POR05 EXHAUST SKID & $\begin{array}{l}\text { WHITEY /SS-65TF16- } \\
\text { LLK }\end{array}$ & NA & NA & $\begin{array}{l}\text { H-2-829116 SHT i H-14: } \\
100867 \text { SHT } 2,18 \text { Item \# } \\
96\end{array}$ & $\overline{N A}$ & GS & $\overline{\mathrm{NA}}$ \\
\hline POR05-VTP.V.202 & $\begin{array}{l}\text { GLYCOL PUMP } 001 \text { DISCHARGE } \\
\text { VALVE }\end{array}$ & POR05 EXHAUST SKID & $\begin{array}{l}\text { WH:ITEY/SS-65TF16- } \\
\text { LLK }\end{array}$ & NA & $\overline{\mathrm{NA}}$ & $\begin{array}{l}\text { H-2-829116 SHT } 1 \text { H-14. } \\
100867 \text { SHT 2, } 18 \text { item \# } \\
96\end{array}$ & $\mathrm{NA}$ & GS & NA \\
\hline POROS-VTP-V-204 & $\begin{array}{l}\text { GLYCOL EXPANSION TANK } 001 \\
\text { VALVE }\end{array}$ & POR05 EXHAUST SKID & $\begin{array}{l}\text { WHITEY/SS-65TF12- } \\
\text { LLK }\end{array}$ & NA & NA & $\begin{array}{l}\text { H-2-829116 SHT } 1 \text { H-14. } \\
100867 \text { SHT 3, } 7,16 \text { Item } \\
\# 124\end{array}$ & & GS & NA \\
\hline POR05-VTP.V.301 & $\begin{array}{l}\text { SAMPLE FLOW CONTROL VALVE } \\
301\end{array}$ & POR05 EXHAUST SKIO & WHITEYISS-63ES8 & NA & NA & \begin{tabular}{|l|}
$\mathrm{H}-2-829116$ SHT 5 H-14- \\
100916 SHT 3 Item \# 20 \\
\end{tabular} & NA & GS & NA \\
\hline POR05-VTP-V-302 & $\begin{array}{l}\text { SAMPLE FLOW CONTROL VALVE } \\
302\end{array}$ & POR05 EXHAUST SKID & WHITEYISS.63ES 8 & NA & NA & $\begin{array}{l}\text { H-2-829116 SHT } 5 \text { H-14- } \\
100916 \text { SHT } 3 \text { Item \# } 20\end{array}$ & $\overline{N A}$ & SS & NA \\
\hline POR05-VTP-V-303 & $\begin{array}{l}\text { SAMPLE VACUUM PUMP } 301 \\
\text { INLET }\end{array}$ & POR05 EXHAUST SKID & NUPRO/SS-XS-SERIES & NA & $\mathrm{NA}$ & $\begin{array}{l}\text { H-2-829116 SHT } 5 \mathrm{H}-14- \\
100916 \text { SHT } 4 \text { Item } \# 63\end{array}$ & NA & GS & $\overline{N A}$ \\
\hline POR05-VTP-V-304 & SAMPLE VACUUM PUMP 301 & POR05 EXHAUST SKID & WHITEY/SS-1RS8-P & NA & NA & $\begin{array}{l}\mathrm{H}-2-829116 \text { SHT } 5 \mathrm{H}-14- \\
100916 \text { SHT } 4 \text { item } \# 62\end{array}$ & NA & GS & $\overline{\mathrm{NA}}$ \\
\hline POR05-VTP.V.305 & $\begin{array}{l}\text { SAMPLE VACUUM PUMP } 301 \\
\text { OUTLET }\end{array}$ & POR05 EXHAUST SKID & WHITEY/SS-45S8-A-P & NA & NA & $\begin{array}{l}\text { H.2.829116 SHT } 5 \text { H-14- } \\
100916 \text { SHT } 4 \text { item \# } 61\end{array}$ & NA & GS & NA \\
\hline POR05-VTP-V-306 & $\begin{array}{l}\text { SAMPLE VACUUM PUMP } 302 \\
\text { INLET }\end{array}$ & POR05 EXHAUST SKID & NUPRO/SS-XS-SERIES & NÁ & NA & $\begin{array}{l}\text { H-2.829116 SHT } 5 \text { H-14- } \\
100916 \text { SHT } 4 \text { Item \# } 63\end{array}$ & NA & GS & NA \\
\hline POR05-VTP-V-307 & SAMPLE VACUUM PUMP 302 & POR05 EXHAUST SKID & WHITEY/SS-1RS8-P & NA & $\mathrm{NA}$ & $\begin{array}{l}\text { H-2-829116 SHT } 5 \text { H-14- } \\
100916 \text { SHT } 4 \text { ltem \# } 62\end{array}$ & NA & GS & $\overline{N A}$ \\
\hline POR0S-VTP.V-308 & $\begin{array}{l}\text { SAMPLE VACUUM PUMP } 302 \\
\text { OUTLET }\end{array}$ & POR05 EXHAUST SKIO & WHITEY/SS-45S8-A-P & NA & NA & $\begin{array}{l}\text { H-2-829116 SHT } 5 \text { H-14. } \\
100916 \text { SHT } 4 \text { Item \# } 61\end{array}$ & NA & GS & NA \\
\hline POROS-VTP-VFD.001 & $\begin{array}{l}\text { VARIABLE FREQUENCY DRIVE } \\
\text { FOR VENT TRAIN EXHAUST FAN } \\
\end{array}$ & POR05 EXHAUST SKID & $\begin{array}{l}\text { ALLEN-BRADLEY/1305- } \\
\text { BA06A }\end{array}$ & NA & 22758 ITEM \# 5 & $\begin{array}{l}\text { H-2-829116 SHT } 5 \text { H-14- } \\
100870 \text { SHT } 1 \text { Item \# } 42\end{array}$ & NA & SS & YES \\
\hline POR05-VTP.XA-101 & $\begin{array}{l}\text { EXHAUST GENERAL FAILURE } \\
\text { (CLEAR BEACON) }\end{array}$ & POR05 EXHAUST SKID & EOWAROS/47C-N5 & NA & $\overline{N A}$ & $\begin{array}{l}\text { H-2-829116 SHT } 1 \text { H-14- } \\
100869 \text { SHT } 1,4 \text { item \# } \\
9\end{array}$ & NA & GS & YES \\
\hline
\end{tabular}




\begin{tabular}{|c|c|c|c|c|c|c|c|c|c|}
\hline EIN & DESCRIPTION & LOCATION & $\begin{array}{l}\text { MANUFACTURER I } \\
\text { MODEL NUMBER }\end{array}$ & SPECIFICATION & $\begin{array}{c}\text { CVI } \\
\text { SUPPLEMENT }\end{array}$ & DRAWING & $\begin{array}{l}\text { MAINTENANCE } \\
\text { PROCEDURE }\end{array}$ & $\begin{array}{l}\text { SAFETY } \\
\text { CLASS }\end{array}$ & $\begin{array}{l}\text { SPARE } \\
\text { PARTS }\end{array}$ \\
\hline POR05-VTP-XA-301 & $\begin{array}{l}\text { EXHAUST HIGH RAD/CAM } \\
\text { FAILURE (REO BEACON) }\end{array}$ & POR05 EXHAUST SKID & $\begin{array}{l}\text { EOWARDS / 52R-N5- } \\
\text { 40WH }\end{array}$ & NA & NA & $\begin{array}{l}\text { H-2-829116 SHT 1 H-14- } \\
100868 \text { SHT 2, } 4 \text { ITEM \# } \\
142\end{array}$ & NA & GS & NA \\
\hline POR05-VTP-XA-302 & $\begin{array}{l}\text { EXHAUSTER BUZZER (CAM) } \\
\text { VIBRATONE }\end{array}$ & POR05 EXHAUST SKIO & $\begin{array}{l}\text { FED SIGNAL } \\
\text { CORP/MODEL: \# A-4 }\end{array}$ & NA & $\overline{N A}$ & $\begin{array}{l}\text { H-2-829116 SHT-1 H-14. } \\
100870-2,4 \text { Item } \# 57\end{array}$ & NA & GS & NA \\
\hline POR0S-VTP-YYC-101 & $\begin{array}{l}\text { PLC FOR PORTABLE EXHAUST } \\
\text { SYSTEM }\end{array}$ & POR05 EXHAUST SKIO & $\begin{array}{l}\text { ALLEN-BRADLEY / 1746- } \\
\text { P4, 1746-L532, 1746- } \\
1012,1746-N I O 41,1746- \\
\text { NI4, 1746-SN, NI04V, } \\
\text { \#2706-M1N1 }\end{array}$ & NA & $\begin{array}{c}22758 \text { ITEM } \# 1, \\
2,3,4,5,6\end{array}$ & $\begin{array}{l}\text { H-14-100870 SHT 1, 2, 3 } \\
\text { ITEM \# } 44,45,46,87,88, \\
90,91,59\end{array}$ & NA & SS & YES \\
\hline & & & & & & & & & \\
\hline & & & & & & & & & \\
\hline & & & & & & & & & \\
\hline & & & & & & & & & \\
\hline & & & & & & & & & \\
\hline & & & & & & & & & \\
\hline & & & & & & & & & \\
\hline & & & & & & & & & \\
\hline & & & & & & & & & \\
\hline & & & & & & & & & \\
\hline & & & & & & & & & \\
\hline & & & & & & & & & \\
\hline & & & & & & & & & \\
\hline & & & & & & & & & \\
\hline & & & & & & & & & \\
\hline & & & & & & & & & \\
\hline & & & & & & & & & \\
\hline & & & & & & & & & \\
\hline & & & & & & & & & \\
\hline & & & & & & & & & \\
\hline & & & & & & & & & \\
\hline & & & & & & & & & \\
\hline & & & & & & & & & \\
\hline & & & & & & & & & \\
\hline & & & & & & & & & \\
\hline & & & & & & & & & \\
\hline & & & & & & & & & \\
\hline & & & & & & & & & \\
\hline & & & & & & & & & \\
\hline & & & & & & & & & \\
\hline & & & & & & & & & \\
\hline & & & & & & & & & \\
\hline
\end{tabular}

